\title{
Archives of Internal Medicine
}

\begin{tabular}{lrr}
\hline \hline VOL. 26 & AUGUST, 1920 & No. 2 \\
\hline
\end{tabular}

\section{BODY WEIGHT IN TWO HUNDRED AND TWENTY- NINE ADULTS}

\author{
WHICH STANDARD IS THE BEST? \\ H. GRAY, M.D., AND J. F. MAYALL \\ BOSTON OXFORD, MASS.
}

PROBLEM

It is rare for clinicians to recognize that medicine, as a whole, profits more by normal standards than by scattered observations of interesting pathologic cases. This is Benedict's judgment, ${ }^{1}$ and Galton's ${ }^{2}$ appears to have been much the same: "Variations of weight are the surest guides to health. . . . Dangerous illnesses may be avoided, and even life preserved." Such illnesses may be outspoken, like malnutrition in children, thyrotoxicosis and tuberculosis; also diabetes and obesity. Or the disease may be insidious, in which case the weight abnormalities are apt to be called defects without obvious cause. Such defects have been alarmingly frequent in recent military examinations. And not only frequent, but surprisingly important. For instance, if we are conservative and consider not the incidence among draftees but only the actual rejections, we find that overweight ranks second among the prominent causes of rejection, as detailed in Table 1.

TABLE 1.-PhYsical Calses of Rejection

\begin{tabular}{|c|c|c|c|}
\hline \multirow{3}{*}{$\begin{array}{l}\text { Causes } \\
\text { of } \\
\text { Rejection } \\
\text { in Order of } \\
\text { Frequency }\end{array}$} & $\begin{array}{l}\text { By Seven Loenl Boards } \\
\text { for the Army }\end{array}$ & $\begin{array}{l}\text { By the Navy and } \\
\text { Marine Corps }\end{array}$ & $\begin{array}{l}\text { By the Total Draft } \\
\text { for the Army }\end{array}$ \\
\hline & No. examined...... 5,611 & No. examined.... 82,592 & No. examined. $2,510,591$ \\
\hline & $\begin{array}{c}\text { Percentage of } \\
\text { Examinees Rejected }\end{array}$ & $\begin{array}{c}\text { Percentage of } \\
\text { Fxaminees Rejected }\end{array}$ & $\begin{array}{c}\text { Percentage of } \\
\text { Examinees Rejected }\end{array}$ \\
\hline $\begin{array}{c}1 \\
2 \\
3 \\
13 \text { etc. }\end{array}$ & 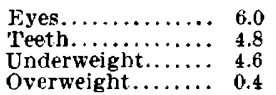 & $\begin{array}{l}\text { Eyes............. } 11.4 \\
\text { Underweight..... } 6.5 \\
\text { Overweight not given }\end{array}$ & $\begin{array}{ll}\text { Heart............. } & 4.3 \\
\text { Underweight....... } & 2.6 \\
& \\
\text { Obesity .......... } & 0.17\end{array}$ \\
\hline
\end{tabular}

The literature furnishes an embarrassing variety of standards by which to judge whether the weight of a given person is within normal

1. Benedict, F. G.: Boston M. \& S. J. 181:109, 117, 118 (July 31), 1919.

2. Galton, F.: Life History Album, London, 1884, p. 2.

3. Fisher, I., and Fisk, E. L.: How to Live, Ed. 15, New York and London, 1919, p. 400, 258.

4. Love, A. G., and Davenport, C. B.: Defects Found in Drafted Men, Printed for Senate Committee on Military Affairs, Washington, 1919, p. 71. 
limits. To enable the physician or statistician to choose between these methods is the main object of this paper. The plan has been to collect measurements and actual weights on a series of normal individuals, then to predict the weights for these men by means of various standards, and finally by comparing these predicted weights with the actual weights to compute the error made by each standard.

\section{INDIVIDUALS OBSERVED}

The first series was abruptly terminated by entry into service of one of the authors, and was, therefore, reported at the time. ${ }^{5}$ It is summarized in Table 7 , together with some new calculations based on the original data. But this earlier series comprised only twenty subjects, and so it was thought desirable to assemble a second group. This was done in the intervals between regular work in the U. S. Army Base Hospital 76, mostly in France, while the extensive computations have been carried out since leaving the service. This second series comprises 229 soldiers, all from the personnel of Base Hospital 76. They were all born in the United States, mostly in Maryland and Pennsylvania. Most of them were inducted at Fort Oglethorpe in June, 1918, and mobilized as this hospital unit at Camp Devens in July, 1919. At that time they were weighed naked, measured and examined physically by the medical officers of the unit, in the hope of excluding some on the ground of possible defects overlooked in the hastier examination at enlistment. Incidentally, the number of positive Wassermanns in 200 tests was only one. The unit sailed from New York August 31, spent about a week in England, and was on duty in Vichy, Allier, France, from the latter part of September until after the time of the second weighing in January, 1919. Some of the original members had meanwhile been separated from the unit, but all who had been weighed twice at the six months' interval are included in this report. Nobody was excluded by preconceived notions on our part as to the typical man. The series is, therefore, unselected, except that males of military age who happen not to fit the army standard are not represented. This fact constitutes a technical defect, as pointed out by Hoffman, but the advantage accrues to a method other than that championed in this paper. Accordingly, we have assumed these to be fair samples of American-born young men, and as such to be suitable controls by which to test the accuracy or error of different methods for predicting weight.

The gist of this test is that Bornhardt's standard is the most accurate, closely followed by the Army and insurance tables; while

5. Gray, H., and Gray, K. M.: Normal Weight, Boston M. \& S. J. 177:894 (Dec. 27) 1917. 
Guthrie's, Broca's and von Noorden's give much less satisfactory results. This is shown graphically in the accompanying chart, and is detailed numerically in Table 9. The casual reader, therefore, may well omit the following minutiae as to method, and skip to the conclusions at the end of the paper.

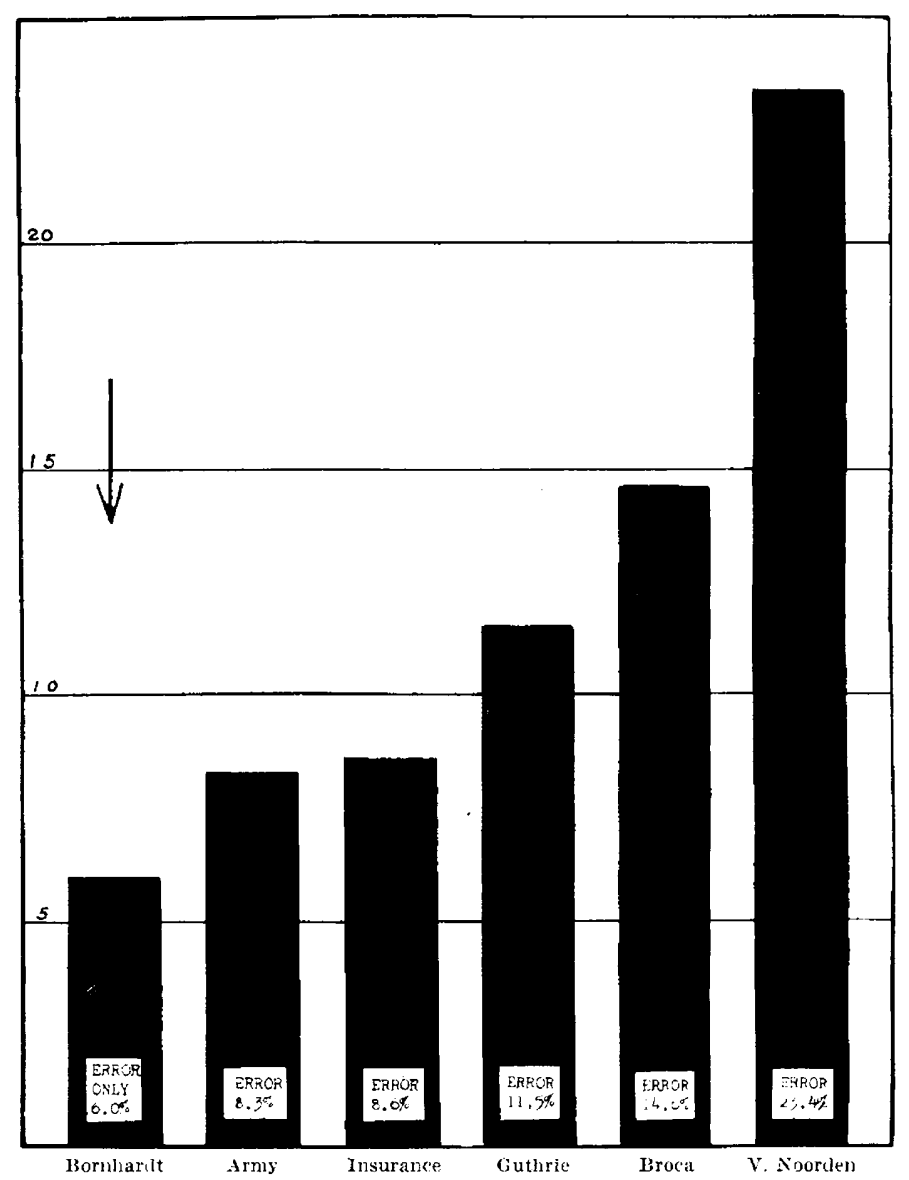

Average error in predicting weight by different standards. Difference between predicted and actual weights, expressed as percentage of the actual weight.

\section{DEFINITIONS}

The actual weight is often represented by such terms as: net weight, weight stripped, weight without clothes. This is the criterion by which we are to choose between various standards in the literature.

The Weight of Clothes.-Nearly every standard except the medicoactuarial is expressed as net weight. In our previous observations ${ }^{5}$ 
men's clothing averaged eight pounds $(3.6 \mathrm{~kg}$.), and heels averaged one inch $(2.5 \mathrm{~cm}$.). By these values the medico-actuarial table, derived from life insurance records, has been adjusted for net weight in making the comparisons both in the earlier series and the present series. It would have been more accurate, however, for us to have subtracted not 8 , but only 6 pounds $(2.7 \mathrm{~kg}$.) from the medico-actuarial table to find the predicted weight, because that table was based on weighings with clothes and shoes, but without coat and vest. If, now, we should correct by an increase of 2 pounds our weights predicted by that table, they would be mathematically more correct, but would make no better showing for that standard, since the predictions already run too high. In practice, when using any other than the insurance table, the average weight of house clothes and shoes for men at all ages, sizes, and seasons may be allowed for by Table 2 . The figures opposite Quetelet's ${ }^{6}$ name were obtained by applying his rule, that clothes average one-eighteenth of the total weight of the male body, to the average weight for the age group here considered, i. e., from 20 to 34 years inclusive (not the average weight for our own group, which is so small relatively and gives 7.8 pounds, i. e., $3.5 \mathrm{~kg}$, but) as computed by us from figures given $^{9}$ for 130,301 men insured in the United States and Canada: 152.1 pounds $(69.1 \mathrm{~kg}$.) with clothes; then clothes come to $152.1 / 18=$ 8.5 pounds $(3.8 \mathrm{~kg}$. $)$.

TABle 2.-Allowance for Mra's Clothes and Shoes*

\begin{tabular}{|c|c|c|}
\hline \multirow{2}{*}{ Writer } & \multicolumn{2}{|c|}{ Weight of Clothes and Shoes } \\
\hline & Kg. & Lbs. \\
\hline 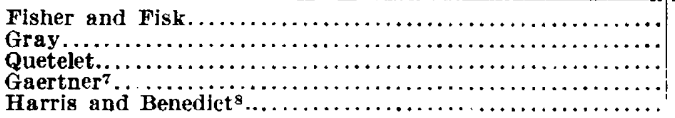 & $\begin{array}{l}3.1 \\
3.6 \\
3.8 \\
4.0 \\
4.0\end{array}$ & $\begin{array}{l}6.9 \\
8.0 \\
8.5 \\
8.8 \\
8.8\end{array}$ \\
\hline
\end{tabular}

* Expressed as kilograms or pounds.

The ideal weight is that accompanied by the healthiest or longest life. Too few people are familiar with the insurance experience ${ }^{3}$ that one should endeavor to keep his weight at the average for his height and for age 30 , the period of full maturity.

6. Quetelet, L. A. J.: Sur l'homme, Paris, 1835, 2:44, or in the first English translation, A Treatise on Man, Edinburgh, 1842, p. 64.

7. Gaertner, G.: Diätetische Entfettungskuren, Leipzig, 1913, pp. 154, 158. 160 ; or in English translation, Reducing Weight Comfortably, Philadelphia, 1914, pp. 278, 286, 291.

8. Harris, J. A., and Benedict, F. G.: A Biometric Study of Basal Metabolism in Man, Carnegie Institute, Washington, Publication No. 279, 1919, pp. v, $2,3,4,7,21,26,163,164,228$.

9. Medico-Actuarial Mortality Investigation. Vol. I, Statistics of Height and Weight of Insured Persons, published by Assn. Life Ins. Directors and Actuarial Soc. of America. N. Y., 1912. pp. 13, 16, 18, 28, 67, 105, 109. 
The average weight, or usual weight, is the familiar arithmetic mean weight, of subjects grouped by age, height, or other more complex methods. In the medico-actuarial investigation the average weight agreed closely with the central weight (also technically called the mode, the most frequent or the typical weight), thus showing the reliability of using the average.

The correct weight may signify either the ideal or the average weight, hence unfortunately it leads to ambiguity.

The normal weight also may be interpreted as either the ideal or the average weight. For children Holt ${ }^{10}$ says that "the average is not to be confused with the normal. There are considerable variations on either side of the average which should be regarded as within the range of normal. The normal is a zone, not a line." Benedict, ${ }^{1}$ too, believes that "for the growing child a gross error has been committed in . . . accepting the average . . . as normal." For adults the insurance committee makes a like statement: "Normal weight for any age and height may vary considerably from the average."

Normal Zone Weight.- This term might be used for the healthy range called "standard lives" by the insurance committee, "the norm of weight" by Gaertner, and more recently emphasized by Holt in the words cited above. The phrase is suitable to express any weight within acceptable limits, but yet not the ideal nor the exact average. The lower boundary of this zone, i. e., the minimal permissible figure beneath which the person is judged underweight, is given by Emerson ${ }^{11}$ as 7 per cent., and by Holt as 10 per cent., both for children. For adults, figures which we have calculated from the 1916 printing of the Army standard give 9 per cent. (ranging from 6 to 13 per cent. according to height), and from the 1919 edition 13 per cent. (ranging from 8 per cent. acceptable below the standard weight for 60 inches, up to 19 per cent. below the standard weight for 74 inches); while the insurance companies regarded lives as standard even down to 15 per cent. underweight.

The standard weight may mean the ideal, or the average, or the zone.

A weight standard is any method, whether table or formula, for predicting weight, as in the title to this paper.

Pathologic Weight, Underweight, Overieight.-Whatever term be used, whatever standard be selected, whatever variation above and beneath the average be accepted as healthy, nearly every writer on obesity or malnutrition starts his reckoning from the predicted weight.

10. Holt, L. E.: Am. J. Dis. Child. 16:359 (Dec.) 1918.

11. Emerson, W. R. P.: Am. J. Dis. Child. 17:251 (April) 1919; Boston M. \& S. I. $181: 139$ (July 31) 1919. 
Predicted weight, calculated weight, estimated weight, expected weight, theoretical weight. This value may be derived from a variety of tables and formulae, of which six are analyzed. It usually corresponds to the average weight.

\section{STANDARDS COMPARED}

While the average weight is affected by various factors, e. g., age, sex, country and locality of birth, height, chest-girth, individual variation or sampling; the weight has in nearly every standard offered been considered in connection with one of the following four periods of life: Birth, preschool age, school age, or adult life. The borders of these four groups, except of course the first, vary somewhat according to different writers, but this is of no concern here. For each of the first three age-groups one table was chosen and reproduced in a previous paper $;^{5}$ while for the last or adult age group six standards were quoted: 'Two tables and four formulas. They were Bornhardt's, the Army's, the medico-actuarial, Guthrie's, Broca's and von Noorden's.

Bornhardt's formula was used in the shape given by Fröhlich, ${ }^{\mathbf{1 2}}$ Vierordt, ${ }^{13}$ Gaertner, ${ }^{7}$ Baer, ${ }^{14}$ Barker ${ }^{15}$ and Vedder, ${ }^{16}$ namely: If $\mathrm{H}$ be the height without heels in centimeters, $C$ the mean chest girth in centimeters as measured over the nipples, W the net weight in kilograms, then the expected weight for the adult of average constitution is: $\mathrm{W}=\mathrm{H} \times \mathrm{C} / 240$.

The Army Table ${ }^{17}$ used in our earlier paper ${ }^{5}$ has been adhered to in the present study, for the sake of uniformity. The editions of "the physical examination standards . . . are . . . all together seven in number," 18 from G. O. 66, A. G. O., April 18, 1910, to the latest and larger table authorized Nov. 8, 1918, and published in Washington, 1919. The changes have been slight.

The Nar'y Table ${ }^{19}$ gives the same weights for the same heights, i. e., from 64 to 73 inches inclusive, as in the edition of the Army table used here.

12. Fröhlich, H.: Allgem. medic. Central. Zeitg. 64:8 (Jan. 2) 1895.

13. Vierordt, H.: Tabellen für Mediziner. Ecl. 3, Jena, 1906, p. 97.

14. Baer, J.: Fettsucht (Obesity), in L. Mohr and R. Staehelin's Handbuch der inneren Medicin, Berlin 4:650, 1912.

15. Barker, L. F.: Clinical Diagnosis of Internal Dis., New York and London 3:794, 1916; or Monographic Medicine 4:794, 1916.

16. Vedder, E. B.: Sanitation for Medical Officers, Medical War Manual No. 1, Ed. 2, Philadelphia, 1918, p. 74.

17. McCain, H. P., Adjutant General, War Department, U. S. A. Recruiting Circular No. 2, Washington, Nov. 1, 1916, i. e.. G. O. 66 W. D.

18. Hoffmann, F. L.: Army Anthropometry, Prudential Press, Newark, N. J. 34: $46,1918$.

19. Bureau of Navigation, U. S. Navy, Circular Relating to the Enlistment of Men, July 20, 1912, also Circular N. M. S. 126,480, October, 1916; also Manual for the Medical Department, U. S. Navy, 1917, par. 2082. 
The Medico-Actuarial Table is not reproduced, being very long and available elsewhere. $\overline{0}, 9,20$

Broca's Formula, so-called, ${ }^{15}$ has also been credited to Quetelet ${ }^{21}$ by some, ${ }^{22}$ to Allaire ${ }^{23}$ and to Robert ${ }^{24}$ by others, ${ }^{7,}{ }^{25}$ and cited without credit by still others. ${ }^{26}$ We have not succeeded in finding the original, despite search of the supposed sources. The formula is uniformly expressed as: $\mathrm{W}$ (in $\mathrm{kg}$.) $=\mathrm{H}$ (in $\mathrm{cm}$.) minus 100 . Bouchard believes the rule to be approximately true for heights between 168 and $175 \mathrm{~cm}$.; but we find, from applying the rule to the 116 men of our series who fall within this restricted range, that the prediction error, without regard to sign, averages 14 per cent. This is not materially better than the average for our whole series which will be seen in Table 8.

Guthrie's Formula, worked out on patients in Professor Barker's clinic, ${ }^{15}$ is: Wt. in pounds $=110+(5.5 \times[$ ht. in inches minus 60$])$.

Von Noorden's Formula is given by Barker as: Wt in $\mathrm{kg} .=\mathrm{ht}$ in $\mathrm{cm}$. $\times 430$ to 480 . If from this statement the constant factor for average weight be inferred to correspond to the mean between these two values we get: $\mathrm{Wt}$ in $\mathrm{kg}$. $=\mathrm{ht}$ in $\mathrm{cm}$. $\times 455$. This interpretation has been applied both in the earlier paper and in this. The results cause regret that we have not been able to see Von Noorden's original statement of his rule.

\section{OBSERVATIONS}

The method of recording measurements was as follows: Weight to the nearest half-kilogram (or pound), height and chest-girth to the nearest centimeter, and age to the nearest year. ${ }^{8,9,17,19,27}$ Harris and Benedicts point out that "A measurement of stature to the nearest centimeter is about the limit of accuracy. To retain tenths of kilograms is . . a degree of refinement . . Finally, when individuals are recorded to the nearest year of age we may remember that they are on the average a quarter of a year older or younger than the age to which they are assigned." Our technic also conformed in many respects to the recommendations of the Committee on Anthropology of the National Research Council. ${ }^{18}$ For height a tape 150 by $1.5 \mathrm{~cm}$. ( $59 \times \%$ in.) was tacked to the wall so that the lower end was $100 \mathrm{~cm}$.

20. Joslin, E. P.: Diabetic Manual, Ed. 2, Philadelphia, 1919, p. 115.

21. Quetelet, L. A. J.: Anthropométrie, Bruxelles, 1871 , p. 178 , and elsewhere. 22. Sah1i, H.: Diagnostic Methods, trans. from German Ed. 5, by N. B. Potter, Ed. 2, Philadelphia, 1911, p. 28. Heckel, F.: Grandes et Petites Obesités, Paris, 1911, p. 105.

23. Allaire: Recenil de mémoires de médecine milit., III e S. 10:161, 1863.

24. Robert: Recetil de mémoires de médecine milit, III e S. 10:171, 1863.

25. Fröhlich, H.: Körpergewicht, in Real-Encyclopädie der ges. Heilk., Ed. A. Eulenburg, Vienna, 1887, 11:199.

26. Bouchard, C.: Traité de Pathologie Générale, Paris, 3:183, 1900.

27. Treasury Dept., Bureau of War Risk Insurance Form $2 \mathrm{~A}$. 
above the floor. The subject stood in bare feet, touching the wall with heels, hips, shoulders and head; and a block with square end was pressed down firmly on the scalp. For chest-girth a similar tape was applied on a level with the nipples and read at full inspiration and full expiration ; midway between these two readings was taken as the mean chest-girth. The above technic is somewhat more precise than that considered adequate by the Medico-Actuarial Committee $:^{9}$ height to the nearest inch. Incidentally, if inches be preferred to centimeters, the inch may well be divided, not into eighths (binary fractions) as in ordinary rules, but into tenths. Similarly, fractions of pounds are better noted as tenths than as sixteenths (ounces). These decimal fractions occupy less space in the chart, are more quickly written down, and are better adapted for averaging and other statistical manipulation, as pointed out by Roberts ${ }^{28}$ long ago.

The basal data obtained by our measurements are reported in Table $\mathbf{j}$, with the subjects arranged according to their actual weights, the lightest first.

METHODS OF STATISTICAL ANALYSIS OR CALCULATIONS

The importance of giving attention to methods considered fussy by some, has been urged by Harris and Benedict in these words: "Data must be . . . interpreted as the statistical constants . . . indicate without any regard to opinions heretofore expressed. In the past, data have been treated almost exclusively by the simple method of averages and graphic representation. . . . By means of the biometric formulas . . . during the past two decades instances of the demonstration of law and order in processes hitherto apparently chaotic have been rapidly multiplying. The reader who is interested in endresults rather than in methods should pass over these features, just as the general biologist must pass over the details of method. . . . in a paper by an organic chemist, realizing that they are essential to the technical development of the subject."

Pearl ${ }^{29}$ more recently wrote a paper "to illustrate by a concrete example, not only the importance, but indeed the absolute necessity of mathematical tests of the validity of results and conclusions, if medicine is ever to measure up the standards of scientific logic and accuracy which prevail in some, at least, of the other branches of biologic and physical science. Many, indeed most, of the problems of practical

28. Roberts, C.: Manual of Anthropometry, London, 1878, p. 26. For example, see Reference 5 in which Table 2 reproduces the American Medical Association's table for children (first published by Crum) with the common fractions translated by us into decimals.

29. Pearl, R.: A Statistical Discussion of the Relative Efficacy of Different Methods of Treating Preumonia, Arch. Int. Med. 24:398 (Oct.) 1919. 
TABLE 3.-Observed Data

\begin{tabular}{|c|c|c|c|c|c|c|c|c|}
\hline \multirow{3}{*}{ Number } & \multirow{3}{*}{ Name } & \multirow{3}{*}{$\begin{array}{c}\text { Age at } \\
\text { Nearest } \\
\text { Birth- } \\
\text { lay, } \\
\text { in } \\
\text { Years } \\
3\end{array}$} & \multirow{2}{*}{\multicolumn{2}{|c|}{$\begin{array}{c}\text { Height } \\
\text { Without } \\
\text { Shoes }\end{array}$}} & \multirow{3}{*}{$\begin{array}{c}\text { Chest } \\
\text { Girth at } \\
\text { Nipple, } \\
\text { Mean, } \\
\text { in } \\
\text { Cm. } \\
6\end{array}$} & Act & Veight, & tripped \\
\hline & & & & & & July, & Jan., & Per Cent. \\
\hline & & & In. & $\begin{array}{c}\mathrm{Cm} . \\
5\end{array}$ & & $\begin{array}{l}\text { in } \\
\text { Lbs. } \\
7\end{array}$ & $\begin{array}{l}\text { ing. } \\
\text { in } \\
\text { Lbs. } \\
8\end{array}$ & $\begin{array}{c}\text { or } \\
\text { Loss (-) } \\
9\end{array}$ \\
\hline 1 & & 25 & 60 & 152 & 88 & 105 & 120 & +14 \\
\hline 2 & J. N. N. & 22 & $6 f$ & 168 & 83 & 107 & 105 & $\begin{array}{r}1 \\
-2 \\
\end{array}$ \\
\hline 3 & J. M. W. & 31 & 65 & 165 & 84 & 109 & $1 \because 6$ & +10 \\
\hline 4 & P. J. S. & 23 & $6 \overline{3}$ & 165 & 84 & 109 & 136 & +25 \\
\hline & M. L. & 24 & 62 & 157 & 80 & 110 & 117 & +6 \\
\hline 6 & s. s. & 25 & 66 & 168 & 82 & 110 & 117 & +6 \\
\hline 7 & M. K. & 22 & 62 & 157 & 83 & 112 & 112 & 0 \\
\hline 8 & J. K. T. & 22 & 61 & 155 & 83 & 113 & 126 & +12 \\
\hline$\hat{\tilde{y}}$ & G. W. & 30 & 65 & 165 & 89 & 114 & 126 & +11 \\
\hline 10 & T. W. S. & 23 & 64 & 163 & 88 & 116 & 122 & +5 \\
\hline 11 & P. C. F. & 25 & 63 & 160 & 84 & 116 & 124 & +7 \\
\hline 12 & A. J. D. & 22 & 62 & 157 & 86 & 116 & $12 \pi$ & +8 \\
\hline 13 & W. L. D. & 28 & 65 & 165 & 86 & 116 & 139 & $\begin{array}{l}7 \\
+20\end{array}$ \\
\hline 14 & F. W. & 22 & 64 & 163 & 90 & 117 & 129 & +10 \\
\hline 15 & J. H. M. & 28 & 63 & 160 & 84 & 118 & 120 & $\begin{array}{l}10 \\
+2\end{array}$ \\
\hline 16 & W. C. W. & 28 & 63 & 160 & 89 & 118 & 126 & $+\overline{7}$ \\
\hline 17 & S. A. & 27 & 62 & $15 \tau$ & 85 & 119 & 125 & +5 \\
\hline 18 & J. S. & 26 & 65 & 165 & 88 & 120 & 120 & 0 \\
\hline 19 & M. J. & 24 & 65 & 165 & 90 & 120 & 121 & +1 \\
\hline 20 & s. $\mathrm{Y}$. & 28 & 64 & 163 & 86 & 121 & 129 & +7 \\
\hline 21 & H. D. & 25 & 68 & 173 & 90 & 121 & 129 & +7 \\
\hline 22 & $\bar{w} \cdot \mathrm{s}$. & 26 & 58 & 147 & 89 & 122 & 118 & -3 \\
\hline 23 & J.P. V. & 26 & 65 & 165 & 89 & 122 & 122 & n \\
\hline 24 & J. P. D. & 27 & 63 & 160 & 90 & 122 & 122 & 0 \\
\hline 25 & L. W. & 27 & 64 & 163 & 90 & 122 & 122 & 0 \\
\hline 26 & G. D. W. & 33 & 66 & 168 & 80 & 122 & 126 & +3 \\
\hline 27 & J. J. M. & 23 & 66 & 168 & 88 & 122 & 133 & +9 \\
\hline 28 & A. L. M. & 28 & 71 & 180 & 85 & 122 & 135 & +11 \\
\hline 29 & J. C. T. & 27 & 66 & 168 & 90 & 123 & 127 & +3 \\
\hline 30 & w. w. w. & $2 \pi$ & 69 & 175 & 90 & 123 & 130 & +6 \\
\hline 31 & c. o. N. & 27 & 68 & 173 & 84 & 123 & 137 & +11 \\
\hline 32 & W. J. W. & 23 & 62 & 157 & 91 & 123 & 140 & +11 \\
\hline 33 & J. F. M. & 33 & 69 & 175 & 85 & .24 & 129 & +4 \\
\hline 34 & M.E.J. & 19 & 69 & 175 & 86 & 124 & 133 & +7 \\
\hline 35 & P. W. E. & 26 & 67 & 170 & 81 & 124 & 139 & +12 \\
\hline 36 & J. A. F. & 24 & $6 i$ & 170 & 88 & 124 & 146 & +18 \\
\hline 37 & J. 0. & 21 & 65 & 165 & 89 & 125 & 132 & $\begin{array}{l}10 \\
+6\end{array}$ \\
\hline 38 & J.A. W. & 25 & 66 & 168 & 93 & 125 & 132 & +6 \\
\hline 39 & L. S. & 22 & $6 \overline{3}$ & 165 & 90 & 125 & 136 & +9 \\
\hline 40 & L. C. B. & 26 & 65 & 165 & 85 & 125 & 144 & +15 \\
\hline 41 & D. E. F. & 24 & 62 & 157 & 89 & $12 t$ & $\begin{array}{l}128 \\
128\end{array}$ & $\begin{array}{r}1 \\
+2\end{array}$ \\
\hline 42 & L. H. W. & 24 & 66 & 168 & 90 & 126 & 131 & +4 \\
\hline 43 & R.T.F. & 23 & 69 & 175 & 89 & 126 & 134 & +6 \\
\hline 44 & P. S. H. & 24 & 69 & 175 & 83 & 126 & 137 & +9 \\
\hline 45 & A. F. S. & 26 & 65 & 165 & 89 & 128 & 132 & +3 \\
\hline 46 & J. w. & 29 & 68 & 173 & 90 & 128 & 134 & +5 \\
\hline 47 & J. B. R. & 23 & $6 \pi$ & $1 \%$ & 80 & 128 & $\begin{array}{l}136 \\
136\end{array}$ & $\begin{array}{l}1 \\
+6\end{array}$ \\
\hline 48 & L. L. H. & 25 & 68 & 173 & 84 & 128 & 141 & +10 \\
\hline 49 & J. K. & 23 & 64 & 163 & 86 & 128 & 141 & +10 \\
\hline 50 & F.A.C. & 30 & 69 & 175 & 90 & 128 & 141 & +10 \\
\hline 51 & A. F. B. & 31 & 69 & 175 & 86 & 128 & 143 & +12 \\
\hline 52 & L. G. M. & 23 & 65 & 165 & 88 & 128 & 146 & +14 \\
\hline 53 & P. P. I. & 32 & $\begin{array}{l}60 \\
69\end{array}$ & 175 & 85 & 130 & $\begin{array}{l}140 \\
142\end{array}$ & $\begin{array}{r}117 \\
+10\end{array}$ \\
\hline 54 & J. M. D. & 23 & 69 & $\mathbf{1 7 5}$ & 85 & 129 & $\begin{array}{l}145 \\
145\end{array}$ & $\begin{array}{r}10 \\
+12 \\
\end{array}$ \\
\hline 55 & B. I. F. & 23 & 64 & 163 & 89 & 129 & 150 & +16 \\
\hline 56 & A. B. R. & 28 & 67 & 170 & 91 & 130 & 130 & 0 \\
\hline 57 & P.J.S. & 23 & 63 & 160 & 85 & 130 & 131 & +1 \\
\hline 58 & R. M. & 23 & 69 & 175 & 88 & 130 & 135 & +4 \\
\hline 59 & O. I. O. & 22 & 63 & 160 & 88 & 130 & 138 & +6 \\
\hline 60 & E. S. & 28 & 66 & 168 & 88 & 130 & 139 & +7 \\
\hline 61 & F.J.F. & 30 & 65 & 165 & 88 & 130 & 140 & $\begin{array}{l}T \\
+8\end{array}$ \\
\hline 62 & P. R. C. & 25 & 68 & 173 & 93 & 130 & 150 & +15 \\
\hline 63 & A. B. B. & 34 & 70 & 178 & 88 & 130 & 154 & +18 \\
\hline 64 & F. S. & $\begin{array}{l}32 \\
24\end{array}$ & 66 & 168 & 86 & 131 & 134 & $\begin{array}{l}100 \\
+2 \\
\end{array}$ \\
\hline 65 & C. M. A. & 24 & 63 & 160 & 88 & 131 & 135 & +3 \\
\hline 66 & V.P. B. & 31 & 71 & 180 & 88 & 131 & 140 & +7 \\
\hline 67 & J. E. J. & 26 & 62 & 157 & 90 & 131 & 142 & +8 \\
\hline 68 & G. F. M. & 26 & 70 & 178 & 88 & 131 & 144 & +10 \\
\hline 69 & W. S. & 22 & 66 & 165 & 90 & 131 & 146 & +11 \\
\hline 70 & o. C.M. & $2 \overline{5}$. & 66 & 168 & 84 & 132 & 142 & +8 \\
\hline 71 & J. F. & 29 & 66 & 168 & 86 & 132 & 151 & +14 \\
\hline 72 & H. R. S. & 21 & 67 & 170 & 87 & 132 & 152 & +15 \\
\hline 73 & M. W. & 27 & 68 & 173 & 91 & 133 & 13.3 & 0 \\
\hline 74 & V. S. D. & 29 & 66 & 168 & 86 & 133 & 134 & +1 \\
\hline 75 & W. D. J. & 23 & 69 & 175 & 89 & 133 & 135 & +2 \\
\hline 76 & F. W. F. & 23 & 67 & 170 & 91 & 133 & 140 & +5 \\
\hline 77 & W. s. & 28 & 65 & 165 & 93 & 133 & 142 & +7 \\
\hline
\end{tabular}


TABLE 3.-Observed Data-Continued

\begin{tabular}{|c|c|c|c|c|c|c|c|c|}
\hline \multirow{3}{*}{ Number } & \multirow{3}{*}{$\begin{array}{l}\text { Name } \\
2\end{array}$} & \multirow{3}{*}{$\begin{array}{c}\text { Age at } \\
\text { Nearest } \\
\text { Birth- } \\
\text { day, } \\
\text { in } \\
\text { Years } \\
3 \\
\end{array}$} & \multirow{2}{*}{\multicolumn{2}{|c|}{$\begin{array}{c}\text { Height } \\
\text { Without } \\
\text { Shoes }\end{array}$}} & \multirow{3}{*}{$\begin{array}{c}\text { Chest } \\
\text { Girth at } \\
\text { Nipple, } \\
\text { Mean, } \\
\text { in } \\
\mathrm{Cm.} \\
6 \\
\end{array}$} & \multicolumn{3}{|c|}{ Actual Weight, Stripped } \\
\hline & & & & & & \multirow{2}{*}{$\begin{array}{c}\text { July, } \\
\text { 1918, } \\
\text { in } \\
\text { Lbs. } \\
7 \\
\end{array}$} & \multirow{2}{*}{$\begin{array}{c}\text { Jan., } \\
\text { 1919. } \\
\text { in } \\
\text { Lbs. } \\
8\end{array}$} & \multirow{2}{*}{$\begin{array}{l}\text { Per Cent. } \\
\text { Gain }(+) \\
\text { or } \\
\text { Loss (-) } \\
\quad 9\end{array}$} \\
\hline & & & $\begin{array}{c}\text { In. } \\
4 \\
\end{array}$ & $\begin{array}{c}\mathrm{Cm} . \\
5\end{array}$ & & & & \\
\hline $\begin{array}{l}78 \\
79\end{array}$ & w. C. B. & 23 & 68 & 173 & 93 & 133 & 142 & \\
\hline & I. P. A. & 26 & 50 & 160 & 91 & 133 & & $\begin{array}{l}+7 \\
+9\end{array}$ \\
\hline $\begin{array}{l}80 \\
81\end{array}$ & A. B. C. & $\begin{array}{l}23 \\
23 \\
28\end{array}$ & 71 & 180 & 83 & 133 & 150 & +13 \\
\hline $\begin{array}{l}81 \\
82\end{array}$ & C. L. M. & 27 & $\begin{array}{l}68 \\
67\end{array}$ & $\begin{array}{l}173 \\
170\end{array}$ & $8 \overline{5}$ & 134 & 144 & +7 \\
\hline 83 & L. $G$. & 23 & $\begin{array}{l}67 \\
66\end{array}$ & 168 & $\begin{array}{l}81 \\
85\end{array}$ & $\begin{array}{l}134 \\
134\end{array}$ & $\begin{array}{l}145 \\
148\end{array}$ & $\begin{array}{l}+8 \\
+10\end{array}$ \\
\hline 84 & J. L. B. & $\begin{array}{l}25 \\
23\end{array}$ & $\pi 1$ & 180 & 85 & $\begin{array}{l}134 \\
134\end{array}$ & $\begin{array}{l}148 \\
152\end{array}$ & $\begin{array}{r}+10 \\
+10\end{array}$ \\
\hline 86 & F.M.S. & 31 & $\begin{array}{l}66 \\
67 \\
67\end{array}$ & $\begin{array}{l}165 \\
170\end{array}$ & $\begin{array}{l}89 \\
89\end{array}$ & $\begin{array}{l}134 \\
135\end{array}$ & $\begin{array}{l}152 \\
133\end{array}$ & $\begin{array}{r}+13 \\
-2\end{array}$ \\
\hline 87 & F. K. & $\begin{array}{l}22 \\
27\end{array}$ & 6 & $\begin{array}{l}173 \\
170\end{array}$ & $\begin{array}{l}89 \\
89\end{array}$ & $\begin{array}{l}13 \overline{7} \\
13 \overline{7}\end{array}$ & $\begin{array}{l}135 \\
137\end{array}$ & $\begin{aligned}-2 \\
0 \\
\end{aligned}$ \\
\hline 89 & F.J.D. & 22 & 67 & $\begin{array}{l}160 \\
170\end{array}$ & $\begin{array}{l}89 \\
82\end{array}$ & $\begin{array}{l}135 \\
135 \\
-135\end{array}$ & $\begin{array}{l}137 \\
139\end{array}$ & $\begin{array}{l}+2 \\
+3\end{array}$ \\
\hline 90 & M. R. & $\begin{array}{l}30 \\
27\end{array}$ & 64 & 163 & 91 & 135 & 139 & $\begin{array}{l}+3 \\
+3 \\
\end{array}$ \\
\hline $\begin{array}{l}91 \\
92\end{array}$ & L. J. C. & 32 & $\begin{array}{l}\infty 6 \\
67\end{array}$ & $\begin{array}{l}168 \\
170\end{array}$ & $\begin{array}{l}86 \\
91\end{array}$ & $\begin{array}{l}135 \\
135\end{array}$ & $\begin{array}{l}141 \\
141\end{array}$ & +4 \\
\hline 93 & W. J. M. & 23 & $\pi$ & 178 & 90 & 135 & $\begin{array}{l}141 \\
148\end{array}$ & $\begin{array}{l}+4 \\
+10 \\
+10\end{array}$ \\
\hline $\begin{array}{l}94 \\
95\end{array}$ & $\begin{array}{l}\text { H. A. U. } \\
\text { F. N B }\end{array}$ & 27 & $\begin{array}{l}70 \\
68\end{array}$ & 178 & 91 & 135 & 148 & $\begin{array}{l}70 \\
+10 \\
+10\end{array}$ \\
\hline 96 & B. J. S. & 27 & 64 & $\begin{array}{l}175 \\
163\end{array}$ & $\begin{array}{l}80 \\
93\end{array}$ & $\begin{array}{l}135 \\
135\end{array}$ & $\begin{array}{l}150 \\
150\end{array}$ & $\begin{array}{l}+11 \\
+11\end{array}$ \\
\hline $\begin{array}{l}97 \\
98\end{array}$ & $\begin{array}{l}\text { E. H. W. } \\
\text { R.0., }\end{array}$ & 22 & 67 & 170 & 87 & 135 & 152 & +13 \\
\hline 99 & $\begin{array}{l}\text { R.O. } \\
\text { J. s. }\end{array}$ & 柴0 & $\frac{71}{65}$ & $\begin{array}{l}180 \\
165\end{array}$ & $\begin{array}{l}55 \\
88\end{array}$ & $\begin{array}{l}135 \\
136\end{array}$ & $\begin{array}{l}153 \\
133\end{array}$ & $\begin{array}{r}+13 \\
+9\end{array}$ \\
\hline 100 & J.P. M. & 29 & 67 & 170 & 93 & 136 & 144 & +2 \\
\hline 101 & W. F. W. & 28 & 65 & 165 & 88 & 136 & 145 & +7 \\
\hline $\begin{array}{l}102 \\
703 \\
703\end{array}$ & $\begin{array}{l}\text { J.A. A. B. } \\
\text { G.S. S. }\end{array}$ & $\begin{array}{l}33 \\
3: i\end{array}$ & 67 & $\begin{array}{l}170 \\
173\end{array}$ & $\begin{array}{l}90 \\
89\end{array}$ & 136 & 149 & +10 \\
\hline 104 & O. E.T. & 23 & 69 & $\begin{array}{l}173 \\
175\end{array}$ & $\begin{array}{l}89 \\
90\end{array}$ & $\begin{array}{l}136 \\
136\end{array}$ & 152 & +12 \\
\hline 105 & F.J. S. & 25 & 69 & 175 & 86 & $\begin{array}{l}136 \\
136\end{array}$ & $\begin{array}{l}152 \\
153\end{array}$ & $\begin{array}{l}+12 \\
+13\end{array}$ \\
\hline $\begin{array}{l}106 \\
107\end{array}$ & $\begin{array}{l}\text { C.J. J.A. } \\
\text { G.D. }\end{array}$ & $\begin{array}{l}27 \\
28\end{array}$ & $\begin{array}{l}68 \\
66\end{array}$ & 173 & 90 & 136 & 156 & $\begin{array}{l}+13 \\
+15 \\
+12\end{array}$ \\
\hline 108 & A. z. & 26 & $\begin{array}{l}66 \\
6 \overline{6}\end{array}$ & $\begin{array}{l}1688 \\
165\end{array}$ & $\begin{array}{l}89 \\
93\end{array}$ & ${ }_{13 i}^{136}$ & 157 & $\begin{array}{r}+15 \\
+15\end{array}$ \\
\hline 109 & S. J. H. & 24 & 69 & $17 \overline{5}$ & 97 & $\begin{array}{l}131 \\
137\end{array}$ & $\begin{array}{l}148 \\
152\end{array}$ & $\begin{array}{l}+8 \\
+11\end{array}$ \\
\hline $\begin{array}{l}110 \\
111\end{array}$ & F. J. F. & $\begin{array}{l}25 \\
25\end{array}$ & $\begin{array}{l}69 \\
68\end{array}$ & 175 & 86 & 137 & 161 & $\begin{array}{l}+18 \\
+18\end{array}$ \\
\hline 112 & S. J. H. & 25 & 67 & $\begin{array}{l}173 \\
170\end{array}$ & 94 & $\begin{array}{l}138 \\
138\end{array}$ & $\begin{array}{l}121 \\
139\end{array}$ & $\begin{array}{l}-12 \\
+12\end{array}$ \\
\hline 113 & J. R. K. & 23 & 67 & 170 & 91 & 138 & $\begin{array}{l}146 \\
146\end{array}$ & $\begin{array}{l}+1 \\
+6\end{array}$ \\
\hline $\begin{array}{l}114 \\
115\end{array}$ & $\begin{array}{l}\text { J.W. } \\
\text { E. S. }\end{array}$ & $\begin{array}{l}23 \\
25 \\
25\end{array}$ & 71 & 180 & 85 & 138 & 150 & $\begin{array}{l}+6 \\
+9\end{array}$ \\
\hline 116 & W. & 25 & $\begin{array}{l}66 \\
66\end{array}$ & $\begin{array}{l}168 \\
168\end{array}$ & 94 & $\begin{array}{l}138 \\
138\end{array}$ & 152 & +10 \\
\hline 117 & C.F.S. & 23 & 68 & 173 & $\begin{array}{l}94 \\
87\end{array}$ & $\begin{array}{l}138 \\
138\end{array}$ & $\begin{array}{l}152 \\
157\end{array}$ & $\begin{array}{l}+10 \\
+14\end{array}$ \\
\hline & E. R. W. & 05 & 68 & 173 & 88 & 139 & 140 & $\begin{array}{l}+14 \\
+14\end{array}$ \\
\hline $\begin{array}{l}119 \\
120\end{array}$ & $\begin{array}{l}\text { J.A. A. } \\
\text { P. H.O. }\end{array}$ & $\begin{array}{l}25 \\
25\end{array}$ & 67 & $\begin{array}{l}170 \\
173\end{array}$ & $\begin{array}{l}89 \\
88\end{array}$ & 139 & 144 & $\begin{array}{l}+1 \\
+4 \\
\end{array}$ \\
\hline 121 & $\mathrm{~J}$ M C & 21 & 70 & $\begin{array}{l}113 \\
183\end{array}$ & 94 & $\begin{array}{l}139 \\
139\end{array}$ & $\begin{array}{l}1+6 \\
152\end{array}$ & $\begin{array}{r}+5 \\
+9 \\
+9\end{array}$ \\
\hline 122 & ${ }_{\mathrm{O}}^{\mathrm{H}}, \mathrm{H} \cdot \mathrm{H}_{\mathrm{T}}$ & 28 & 68 & 173 & 83 & $\begin{array}{l}108 \\
140\end{array}$ & $\begin{array}{l}152 \\
132\end{array}$ & $\begin{array}{l}+9 \\
\pm 6\end{array}$ \\
\hline $\begin{array}{l}123 \\
124\end{array}$ & $\begin{array}{l}\text { D. K. T. } \\
\text { R. E. }\end{array}$ & $\begin{array}{l}18 \\
24\end{array}$ & 68 & $\begin{array}{l}173 \\
178\end{array}$ & 86 & 140 & 137 & $\begin{array}{l}-6 \\
-2\end{array}$ \\
\hline 1325 & R. E. H. & 21 & 69 & 175 & $\begin{array}{l}91 \\
86\end{array}$ & $\begin{array}{l}140 \\
140\end{array}$ & $\begin{array}{l}140 \\
145\end{array}$ & $\begin{array}{r}0 \\
+4\end{array}$ \\
\hline 726 & A. C. W. & 28 & 70 & 178 & 87 & $\begin{array}{l}140 \\
140\end{array}$ & $\begin{array}{l}145 \\
142\end{array}$ & $\begin{array}{l}+4 \\
+9\end{array}$ \\
\hline 127 & W. N. S. & $\frac{26}{38}$ & 50 & 178 & 88 & 140 & 154 & $\begin{array}{l}+9 \\
+10\end{array}$ \\
\hline $1 \geq 8$ & A. F.S. & $\frac{28}{31}$ & 67 & $\begin{array}{l}170 \\
180\end{array}$ & 92 & 140 & 174 & $\begin{array}{l}+24 \\
+24\end{array}$ \\
\hline 129 & G. $\mathbf{W} \cdot \mathbf{K}$. & 31 & 72 & 183 & 89 & 141 & 152 & $\begin{array}{r}24 \\
+8 \\
\end{array}$ \\
\hline $\begin{array}{l}130 \\
131\end{array}$ & 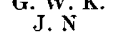 & $\begin{array}{l}24 \\
24\end{array}$ & 68 & 180 & 91 & 141 & 158 & $+3 \%$ \\
\hline $\mathrm{j} 32$ & J. A & 28 & 60 & 165 & 91 & 141 & 168 & +19 \\
\hline 133 & c. $T$. & 28 & $i_{1}$ & 180 & 91 & $\begin{array}{l}142 \\
142\end{array}$ & $\begin{array}{l}135 \\
142\end{array}$ & -5 \\
\hline 134 & P. K. & 26 & 69 & 175 & 91 & 142 & 144 & $\begin{array}{r}0 \\
+2 \\
2\end{array}$ \\
\hline & H. F. G. & 24 & 63 & 160 & 94 & 142 & 144 & $\begin{array}{l}+2 \\
+2 \\
+2\end{array}$ \\
\hline $\begin{array}{l}136 \\
137\end{array}$ & L. A. R. $\mathbf{P}$. & 28 & 72 & $\begin{array}{l}183 \\
183\end{array}$ & 91 & 142 & 153 & $\begin{array}{l}+2 \\
+8\end{array}$ \\
\hline 138 & M.J. R. & $\begin{array}{l}28 \\
28\end{array}$ & 68 & 186 & $\begin{array}{l}91 \\
86\end{array}$ & $\begin{array}{l}142 \\
143\end{array}$ & $\begin{array}{l}153 \\
138\end{array}$ & $\begin{array}{l}+8 \\
+8\end{array}$ \\
\hline 139 & J. S. & 27 & 67 & $1 \% 0$ & 90 & $\begin{array}{l}143 \\
143\end{array}$ & $\begin{array}{l}138 \\
142\end{array}$ & -3 \\
\hline 140 & w. c.s. & 30 & 66 & 1tis: & 83 & 143 & 150 & $\begin{array}{l}-1 \\
+5\end{array}$ \\
\hline 141 & J. J. L. & 22 & io & 178 & 91 & 143 & $15 \mathrm{~T}$ & +6 \\
\hline 142 & A. W. H. & 28 & 72 & 183 & 91 & 143 & 152 & +6 \\
\hline $\begin{array}{l}143 \\
144\end{array}$ & $\stackrel{P}{P} F_{\dot{D}}$ & 28 & 75 & $\begin{array}{l}191 \\
780\end{array}$ & 94 & 143 & 155 & +8 \\
\hline $\begin{array}{l}144 \\
145 \\
\end{array}$ & G.H.S. & 23 & 64 & $\begin{array}{l}\frac{180}{163} \\
163\end{array}$ & $\begin{array}{l}91 \\
90\end{array}$ & $\begin{array}{l}143 \\
144\end{array}$ & $\begin{array}{l}157 \\
143\end{array}$ & $\begin{array}{l}+10 \\
-1\end{array}$ \\
\hline 146 & J.W. T. & 31 & 69 & 175 & 93 & 144 & 144 & 0 \\
\hline 147 & K. R. & 23 & 66 & 168 & 98 & 144 & 156 & +8 \\
\hline 148 & w. H. M. & 23 & 71 & 180 & 90 & 144 & 164 & +14 \\
\hline 149 & $\begin{array}{l}\text { J. C. M. } \\
\text { v. v. }\end{array}$ & 25 & 71 & 180 & $\varepsilon 6$ & 144 & 165 & +15 \\
\hline $\begin{array}{l}150 \\
151\end{array}$ & $\begin{array}{l}\text { v. v. } \dot{0} \text {. } \\
\text { L. D. }\end{array}$ & $\begin{array}{l}26 \\
25\end{array}$ & $\begin{array}{l}63 \\
70\end{array}$ & $\begin{array}{l}160 \\
178\end{array}$ & 99 & 145 & 146 & +1 \\
\hline $\begin{array}{l}151 \\
152\end{array}$ & L. D. O. & 25 & 69 & 178 & 91 & 145 & 147 & +1 \\
\hline $\begin{array}{l}152 \\
153\end{array}$ & J. W. & $\begin{array}{l}28 \\
24 \\
24\end{array}$ & $\begin{array}{l}69 \\
69 \text {. }\end{array}$ & $\begin{array}{l}170 \\
175\end{array}$ & $\begin{array}{l}90 \\
94\end{array}$ & $\begin{array}{l}145 \\
145\end{array}$ & $\begin{array}{l}159 \\
179\end{array}$ & $\begin{array}{l}+10 \\
+23\end{array}$ \\
\hline 154 & W. G. L. & 23 & 67 & 170 & 93 & 146 & 161 & +10 \\
\hline
\end{tabular}


TABLE 3.-Observed Data-Continued

\begin{tabular}{|c|c|c|c|c|c|c|c|c|}
\hline \multirow{3}{*}{ Number } & \multirow{3}{*}{ Name } & \multirow{3}{*}{$\begin{array}{c}\text { Age at } \\
\text { Nearest } \\
\text { Birth- } \\
\text { day, } \\
\text { in } \\
\text { Years } \\
3\end{array}$} & \multirow{2}{*}{\multicolumn{2}{|c|}{$\begin{array}{c}\text { Height } \\
\text { Without } \\
\text { Shoes }\end{array}$}} & \multirow{3}{*}{$\begin{array}{c}\text { Chest } \\
\text { Girth at } \\
\text { Nipple, } \\
\text { Mean, } \\
\text { in } \\
\text { Cm. } \\
6\end{array}$} & \multicolumn{3}{|c|}{ Actuai Weight, Stripued } \\
\hline & & & & & & \multirow{2}{*}{$\begin{array}{c}\text { July } \\
1918, \\
\text { in } \\
\text { Lbs. } \\
7\end{array}$} & \multirow{2}{*}{$\begin{array}{c}\text { Jan., } \\
1919 . \\
\text { in } \\
\text { Lbs. } \\
8\end{array}$} & \multirow{2}{*}{$\begin{array}{l}\text { Per Cent. } \\
\text { Gain }(-) \\
\text { or } \\
\text { Loss (-) } \\
\quad 9\end{array}$} \\
\hline & & & $\begin{array}{c}\text { In. } \\
4\end{array}$ & $\mathrm{Cm}$. & & & & \\
\hline 155 & J. J. K. & 26 & 66 & 168 & 93 & $14 \bar{i}$ & 148 & +1 \\
\hline $\begin{array}{l}156 \\
157\end{array}$ & H. W. T. & 29 & 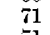 & 180 & 91 & $14 \bar{\tau}$ & 157 & $\begin{array}{l}+1 \\
+7\end{array}$ \\
\hline $\begin{array}{l}157 \\
158\end{array}$ & C. D. K. & 24 & $\pi$ & 180 & 91 & 147 & 164 & +12 \\
\hline 159 & G. S. & 23 & Gî & 170 & ${ }_{91}^{91}$ & $\begin{array}{l}148 \\
148\end{array}$ & $\begin{array}{l}155 \\
118\end{array}$ & +5 \\
\hline 160 & W. D. L. & 26 & 71 & 180 & 91 & 148 & 149 & $\begin{array}{r}0 \\
+1\end{array}$ \\
\hline${ }_{162}^{161}$ & P. J. M. & $\begin{array}{l}23 \\
23 \\
23\end{array}$ & 67 & 170 & 89 & 148 & 15 & $\begin{array}{l}+2 \\
+6 \\
\end{array}$ \\
\hline $\begin{array}{l}162 \\
163\end{array}-x-2$ & $\begin{array}{l}\text { M.N.K. } \\
\text { G. E. K. }\end{array}$ & ${ }_{30}^{23}$ & $\begin{array}{l}69 \\
67\end{array}$ & $\begin{array}{l}175 \\
170\end{array}$ & $\begin{array}{l}86 \\
98\end{array}$ & $\begin{array}{l}148 \\
148\end{array}$ & 170 & +15 \\
\hline 164 & J. B. R. & 24 & 69 & 175 & 88 & 148 & 178 & $\begin{array}{l}+15 \\
+20\end{array}$ \\
\hline $\begin{array}{l}165 \\
166\end{array}$ & R. L. P. & 25 & 70 & 178 & 91 & 149 & 155 & +4 \\
\hline $\begin{array}{l}166 \\
167\end{array}$ & w.s. & $\begin{array}{l}25 \\
2 . \overline{3}\end{array}$ & $\begin{array}{l}68 \\
63\end{array}$ & $\begin{array}{l}173 \\
160\end{array}$ & $\begin{array}{l}89 \\
94\end{array}$ & $\begin{array}{l}149 \\
150\end{array}$ & 162 & $\begin{array}{l}+9 \\
+9 \\
+6\end{array}$ \\
\hline 168 & J. S. & 30 & 69 & 175 & 95 & 150 & 168 & $\begin{array}{l}+6 \\
+12\end{array}$ \\
\hline 169 & F.S. W. & 25 & & 178 & 94 & 150 & 157 & $\begin{array}{l}+12 \\
+5\end{array}$ \\
\hline $\begin{array}{l}170 \\
171\end{array}$ & $\begin{array}{l}\text { D. L. H. } \\
\text { C. J. M. }\end{array}$ & ${ }_{31}^{31}$ & ז1 & $\begin{array}{l}180 \\
173\end{array}$ & 90 & $\begin{array}{l}150 \\
150\end{array}$ & 157 & $\begin{array}{r}+5 \\
+5 \\
\end{array}$ \\
\hline 172 & J.s. & $\begin{array}{l}31 \\
28\end{array}$ & $\begin{array}{l}68 \\
70\end{array}-20$ & $\begin{array}{l}173 \\
178\end{array}$ & 85 & $\begin{array}{l}150 \\
150\end{array}$ & $\begin{array}{l}164 \\
142\end{array}$ & $\begin{array}{r}+9 \\
+5\end{array}$ \\
\hline 173 & н. $0 . \mathrm{W}$. & 29 & 67 & 170 & 95 & 150 & 169 & $\begin{array}{r}-5 \\
+13\end{array}$ \\
\hline${ }_{175}^{174}$ & S. $\mathbf{w} \dot{.}$ & 28 & 68 & ${ }_{173}^{173}$ & 94 & 151 & & $\begin{array}{l}+11 \\
+11\end{array}$ \\
\hline $\begin{array}{l}175 \\
176\end{array}$ & R.T. & $\begin{array}{l}26 \\
22 \\
22\end{array}$ & $\begin{array}{l}68 \\
62\end{array}$ & $\begin{array}{l}17 \overline{3} \\
1 \overline{\bar{y}}\end{array}$ & $\begin{array}{l}9 ; 3 \\
92\end{array}$ & $\begin{array}{l}151 \\
152\end{array}$ & $\begin{array}{l}177 \\
155\end{array}$ & $\begin{array}{l}+17 \\
+2\end{array}$ \\
\hline 177 & F. P. A. & 29 & 70 & $\begin{array}{l}101 \\
178\end{array}$ & 94 & 152 & $\begin{array}{l}162 \\
162\end{array}$ & $\begin{array}{l}+2 \\
+7\end{array}$ \\
\hline 178 & C. A. E. & 23 & 70 & 178 & 89 & 152 & 165 & $\begin{array}{l}+9 \\
+9\end{array}$ \\
\hline 179 & H. S. F. & 29 & 70 & 178 & 99 & 152 & 169 & +11 \\
\hline $\begin{array}{l}180 \\
181\end{array}$ & $\mathbf{E}, \mathbf{F} . \dot{P}$ & 24 & $\begin{array}{l}68 \\
78 \\
78\end{array}$ & $\begin{array}{l}173 \\
178\end{array}$ & $\begin{array}{l}97 \\
97 \\
93\end{array}$ & 152 & 169 & +11 \\
\hline 182 & F.D.S. & 25 & 70 & 178 & 等 & $\begin{array}{l}1.153 \\
153\end{array}$ & 171 & $\begin{array}{l}+8 \\
+12\end{array}$ \\
\hline 183 & L. R. & 29 & 66 & 168 & $9_{i}$ & 154 & 170 & $\begin{array}{l}+12 \\
+10\end{array}$ \\
\hline 184 & C. 1 & 22 & 64 & 163 & 97 & 155 & 157 & $\begin{array}{l}+0 \\
+1\end{array}$ \\
\hline 185 & M. A. M. & 24. & 74 & 188 & 88 & 155 & 159 & +3 \\
\hline${ }_{187}^{186}$ & H. A. & 年 25 & 68 & $\begin{array}{l}173 \\
180\end{array}$ & 89 & ${ }_{156}^{155}$ & 164 & +6 \\
\hline $\begin{array}{l}187 \\
188\end{array}$ & $\begin{array}{l}\text { E. H. B. } \\
\text { D. }\end{array}$ & $\begin{array}{l}28 \\
24\end{array}$ & $\pi$ & $\begin{array}{l}180 \\
180\end{array}$ & $\begin{array}{l}90 \\
89\end{array}$ & $\begin{array}{l}156 \\
156\end{array}$ & $\begin{array}{l}157 \\
160\end{array}$ & $\begin{array}{l}+1 \\
+3\end{array}$ \\
\hline${ }_{189}^{189}$ & T. J. C. & 31 & 64 & 163 & 95 & 156 & 166 & +6 \\
\hline 190 & 矛. & 31 & ${ }^{71}$ & ${ }_{185}$ & 98 & 156 & 150 & $\begin{array}{l}+9 \\
\end{array}$ \\
\hline $\begin{array}{l}191 \\
192\end{array}$ & E. A. B & $\begin{array}{l}23 \\
24 \\
24\end{array}$ & $\begin{array}{l}69 \\
69\end{array}$ & $\begin{array}{l}175 \\
175\end{array}$ & $\begin{array}{l}89 \\
93\end{array}$ & $\begin{array}{l}156 \\
156\end{array}$ & $\begin{array}{l}171 \\
176\end{array}$ & $\begin{array}{l}+10 \\
+13\end{array}$ \\
\hline 193 & $R_{i} M_{i}$ & $\begin{array}{l}28 \\
28\end{array}$ & 67 & 170 & 96 & 158 & 146 & -8 \\
\hline 194 & D. E. R. & ${ }_{97}^{28}$ & 70 & 178 & 86 & 158 & 160 & +1 \\
\hline $\begin{array}{l}198 \\
196\end{array}$ & $\begin{array}{l}\text { W. } \\
\text { W. B. }\end{array}$ & $\begin{array}{l}27 \\
20\end{array}$ & $\begin{array}{l}70 \\
68\end{array}$ & $\begin{array}{l}178 \\
173\end{array}$ & $\begin{array}{l}90 \\
94\end{array}$ & $\begin{array}{l}158 \\
158\end{array}$ & $\begin{array}{l}161 \\
165\end{array}$ & $\begin{array}{l}+2 \\
+4\end{array}$ \\
\hline 197 & J. J. K. & 29 & 68 & 173 & 94 & 158 & 172 & $\begin{array}{r}2 \\
+9\end{array}$ \\
\hline 198 & J.B. & ${ }_{98}^{29}$ & 68 & $\begin{array}{l}173 \\
189\end{array}$ & $\begin{array}{l}99 \\
85\end{array}$ & 158 & 174 & +10 \\
\hline $\begin{array}{l}199 \\
200\end{array}$ & R.E.S. & 尊3 & 68 & $\begin{array}{l}183 \\
173\end{array}$ & $\begin{array}{l}85 \\
95\end{array}$ & $\begin{array}{l}160 \\
160\end{array}$ & $\begin{array}{l}153 \\
155\end{array}$ & $\begin{array}{l}-4 \\
-3\end{array}$ \\
\hline 201 & o. $\mathbf{H}, \mathbf{w}$ & 31 & 66 & 168 & 95 & 160 & 175 & +9 \\
\hline $\begin{array}{l}202 \\
203\end{array}$ & w.M. G. & ${ }_{94}^{27}$ & $\begin{array}{l}69 \\
71\end{array}$ & 175 & 95 & 160 & 175 & $\begin{array}{r}+9 \\
\end{array}$ \\
\hline 204 & J. D. G. & ${ }_{23}^{27}$ & 72 & $\begin{array}{l}180 \\
183\end{array}$ & $\begin{array}{l}90 \\
94\end{array}$ & $\begin{array}{l}161 \\
161\end{array}$ & $\begin{array}{l}179 \\
194\end{array}$ & $\begin{array}{l}+11 \\
+20\end{array}$ \\
\hline 205 & F. E. C. & 23 & 69 & 175 & 101 & 162 & 173 & +7 \\
\hline 206 & A.s.s. & 28 & 68 & $\begin{array}{l}173 \\
185\end{array}$ & 84 & 163 & 152 & 77 \\
\hline 208 & P. S. K. & 25 & $\begin{array}{l}16 \\
68\end{array}$ & $\begin{array}{l}100 \\
173\end{array}$ & $\begin{array}{l}93 \\
98\end{array}$ & 164 & $\begin{array}{l}178 \\
170\end{array}$ & $\begin{array}{r}+3 \\
+4 \\
+4\end{array}$ \\
\hline 209 & V. P. N. & 26 & 74 & 188 & 91 & 164 & 183 & $\begin{array}{r}+12 \\
\end{array}$ \\
\hline 210 & G.J.S. & 28 & 68 & 173 & 87 & 165 & 162 & -18 \\
\hline $\begin{array}{l}211 \\
.212\end{array}$ & W. F.R. & $\begin{array}{l}26 \\
25\end{array}$ & $\begin{array}{l}69 \\
71\end{array}$ & $\begin{array}{l}175 \\
180\end{array}$ & 㽬 & $\begin{array}{l}166 \\
167\end{array}$ & $\begin{array}{l}183 \\
168\end{array}$ & $\begin{array}{l}+10 \\
+1 \\
+1\end{array}$ \\
\hline 213 & T. J. D. & 29 & 69 & 175 & 91 & 167 & 170 & $\begin{array}{l}1 \\
+2\end{array}$ \\
\hline 214 & H. C. L. & $2 \pi$ & 67 & 170 & $9 \mathbf{a}$ & 168 & 177 & +5 \\
\hline & C. E. B. & 26 & 67 & 170 & 94 & 168 & 179 & +7 \\
\hline 216 & A. A. R. & 24 & 67 & 1 to & 96 & 169 & 166 & -2 \\
\hline 217 & Ј. К. Z. & 28 & 72 & 183 & 101 & 170 & 186 & +9 \\
\hline${ }_{219}^{218}$ & W. & ${ }_{29}^{23}$ & 70 & $\begin{array}{l}180 \\
178\end{array}$ & $\begin{array}{l}106 \\
102\end{array}$ & 171 & $\begin{array}{l}183 \\
168\end{array}$ & $\begin{array}{r}+7 \\
-2\end{array}$ \\
\hline 220 & M. W H. & 27 & 69 & 175 & 102 & 173 & 173 & 0 \\
\hline 221 & I. $\mathbf{L}$. & 31 & 65 & 165 & 99 & 174 & 180 & +3 \\
\hline 222 & $\begin{array}{l}\text { E. S. S. } \\
\text { T. J. }\end{array}$ & $\begin{array}{l}22 \\
29\end{array}$ & $\begin{array}{l}68 \\
69\end{array}$ & $\begin{array}{l}173 \\
175\end{array}$ & $\begin{array}{l}70 \\
99\end{array}$ & $\begin{array}{l}175 \\
175\end{array}$ & $\begin{array}{l}162 \\
176\end{array}$ & 77 \\
\hline 224 & A. L. A. & 30 & 72 & 183 & 98 & 175 & 190 & $\begin{array}{l}+ \\
+\end{array}$ \\
\hline 225 & J. C. $\mathbf{M}$. & 25 & 72 & 183 & 95 & 176 & 185 & +5 \\
\hline 22 & W. F.S. & 30 & 65 & 165 & 96 & 179 & 184 & +3 \\
\hline 22 & F. J. R. & 30 & 71 & 180 & 95 & 180 & 174 & -3 \\
\hline \multirow{2}{*}{$\begin{array}{l}228 \\
229\end{array}$} & E. E. $\mathbf{W}$. & $\begin{array}{l}30 \\
30\end{array}$ & 71 & 180 & $\begin{array}{r}93 \\
102\end{array}$ & $\begin{array}{l}190 \\
193\end{array}$ & 185 & -3 \\
\hline & Avyerage & 26 & 67 & 177 & 90 & 141 & 150 & +7 \\
\hline & & & & & & & & +7 \\
\hline
\end{tabular}


medicine in a broad sense are either essentially statistical problems, or their statistical phase is a vitally important one in reaching correct conclusions."

Dreyer, ${ }^{30}$ and others, ${ }^{31}$ have also recently urged greater care as to the method of collecting and presenting facts.

Prediction Error.-When the weight of an individual is predicted by any method the value may be identical with the observed weight, but in general deviates or errs somewhat from it. This error may be either above or below the actual weight, i. e., either positive or negative in sign; and in consequence it tells us whether the predictions made by a given method are on the whole too large or too small. Since we are in this case testing methods of prediction against actual observation we have taken the differences: calculated weight less actual weight, or $\mathrm{PW}-\mathrm{W}=\mathrm{D}$. This difference $\mathrm{D}$ is then reduced from pounds or kilograms to its percentage of the actual weight, plus or minus, i. e.,

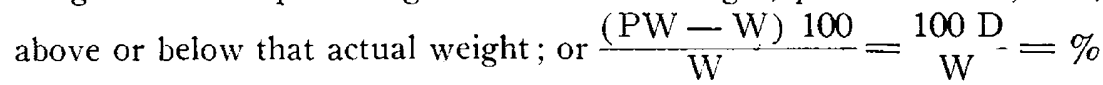
prediction error $=\mathrm{E}$. This percentage prediction error is our common unit on which all further discussion is based hereafter. For example, in Table 4, Bornhardt's method applied to Case 1 gives: $P W=55.9 \mathrm{~kg}$. (123 pounds), $\mathrm{W}=47.7 \mathrm{~kg}$. (105 pounds), hence $\mathrm{D}=8.2 \mathrm{~kg}$. (18 pounds), from which by substitution in the above formula we find the prediction error is $8.2 / 47.7=17$ per cent., or $18 / 105=17$ per cent. In other words, this value $\mathrm{E}$ will be the same no matter whether our observed data have been recorded in metric or customary units, though ordinarily of course all one's data are recorded in the same unit. The error for each individual has been calculated only to the nearest integral, while the average error for each standard is given to about 1 per cent.

The reasons for this percentile manner of expression are:

1. When comparing the varying errors obtained by applying the same formula to the 229 different subjects, percentage errors are always comparable, whereas kilogram or pound errors are often not comparable owing to the subjects' varying weights.

2. When comparing the average of these 229 errors by one formula with the average of the errors by a second formula on the same 229 cases, percentage errors are always comparable, whereas kilogram or pound errors are often not comparable because the two formulas are expressed in different units: one metric, the second English.

30. Dreyer, G., and Walker, E. W. A.: Iatro-mathematics, in Contributions to Medical and Biological Research. Dedicated to Sir William Osler, New York, 1: $40,1919$.

31. Editorial, J. A. M. A. 73:1531 (Nov. 15) 1919; also, Kilgore, E. S.: J. A. M. A. 75:86 (July 10) 1920 . 
3. Furthermore, as will be clearer further on, the expression of the errors as percentages automatically determines limits of the class intervals convenient for tabulating the frequency of each of the different sized errors, for reckoning the average of the 229 errors, or their standard-deviation, or their coefficient of variation.

Analysis of the error, to determine the relative precision of prediction by different methods, is carried out by the application of the following criteria or constants :

1. The frequency distribution of error.

2. The average error with regard to sign, i. e., the algebraic mean.

3 . The average error without regard to sign, i. e., the arithmetical mean.

4. The standard error, i. e., the square root of the sum of the squares of the errors.

5. The range between the maximum error and the minimum error.

6. The standard deviation of the average error, i. e., the square root of the sum of the squares of the deviations of each of the 229 errors from the average error.

7. The coefficient of variation.

The next step is to summarize the errors in the predictions by each method, as given in Table 4, into a frequency-distribution table, Table 5, in conformity with the dictum: "In all cases of published work the figures of the frequency-distribution should be given; they are absolutely fundamental." 32

Calculation of the Mean Error.-Method I (Yule ${ }^{32}$ ): From frequency distribution Table 5 .

Let $A=$ an arbitrary value or guess at approximate mean.

Let $\mathrm{M}=$ true mean.

Let $\mathrm{d}=$ difference between approximate and the true mean $=\mathrm{M}-\mathrm{A}=\mathrm{Sum}$ f. $z / \mathrm{N}$.

Then by substitution of figures from Table 5 .

$$
\mathrm{d}=\frac{\operatorname{Sum}(\mathrm{f} . \mathrm{z})}{\mathrm{N}}=\frac{+453-452}{229}=\frac{+1}{229}=0.0044 \text { class-intervals. }
$$

Now in making the frequency Table 5 , each class-interval was taken as $=1$ per cent. error.

$$
\begin{gathered}
\therefore \mathrm{d}=0.0044 \text { per cent. } \\
\mathrm{M}=\mathrm{A}+\mathrm{d}=6+0.0044=6.0044 \text { per cent. }
\end{gathered}
$$

Method II (Harris and Benedict): From basal Table 3.

$$
\mathrm{V}=\frac{\mathrm{Sum} E}{\mathrm{~N}}=\frac{1374}{229}=6.00 \text { per cent. }
$$

32. Yule, G. U.: Introduction to the Theory of Statistics, Ed. 5, London, 1919 , pp. 79, 112, 133, 141, 144, 153. 
TABLE 4.-Method of Computing Prediction Error; with Examples from the Application of Bornhardt's Standard to the Series

of 229 MaLes

\begin{tabular}{|c|c|c|c|c|c|}
\hline \multirow{4}{*}{. } & \multirow{4}{*}{$\begin{array}{c}\text { Case } \\
\text { Number }\end{array}$} & \multicolumn{4}{|c|}{ Prediction (Standard: Bornhardt's Formula) } \\
\hline & & $\begin{array}{c}\text { Predicted } \\
\text { Weight }\end{array}$ & $\begin{array}{c}\text { Difference } \\
\text { of Predicted } \\
\text { Weight from } \\
\text { Actual Weight, } \\
\text { Too High (+) } \\
\text { Too Low (-) }\end{array}$ & $\begin{array}{c}\text { Percentile } \\
\text { Prediction } \\
\text { Error, } \\
\text { Prediction } \\
\text { Error, or } \\
\text { Simply Frror }\end{array}$ & $\begin{array}{l}\text { Error } \\
\text { Squared }\end{array}$ \\
\hline & & PW & $P W-W=D$ & $\frac{\mathrm{D}}{\mathrm{W}}=\mathrm{E}$ & $\mathrm{E}^{2}$ \\
\hline & & Kg. or Lbs. & Kg. or Lbs. & $\begin{array}{l}\text { Per Cent. } \\
\text { of Actual } \\
\text { Weight } W\end{array}$ & \\
\hline & $\begin{array}{c}1 \\
2 \\
---- \\
229\end{array}$ & $\begin{array}{c}55.9 \\
127 \\
179 \\
\end{array}$ & $\begin{array}{c}+8.2 \\
+20 \\
----- \\
-14\end{array}$ & $\begin{array}{c}+17 \\
+19 \\
---- \\
-7 \\
\end{array}$ & $\begin{array}{r}289 \\
361 \\
---- \\
49\end{array}$ \\
\hline Arithmetic sum........... & $\ldots \ldots \ldots$ & $\ldots \ldots \ldots \ldots$ & n.............. & 1,374 & 14,283 \\
\hline Mean error................ & n.......... & $\ldots \ldots \ldots+\cdots$ & $\ldots \ldots \ldots \ldots \ldots$ & $6.0 \%$ & 62.37 \\
\hline Root-mean-square-error.. & $\ldots \ldots \cdots \cdots$ & $\ldots \ldots \ldots+\cdots$ & $\ldots \ldots \ldots \ldots \ldots \ldots \ldots$ & $\ldots \ldots+\cdots \cdots+\cdots$ & $7.89 \%$ \\
\hline Range of error............. & ............ & ............... & $\begin{array}{c}0 \text { to }+20 \text { and } \\
-64 \mathrm{lbs} \text {. }\end{array}$ & $\begin{array}{c}0 \text { to }+19 \text { and } \\
-36 \%\end{array}$ & 0 to 1,296 \\
\hline Standard deviation.... & ..... & $\cdots$ & $\cdots$ & $5.14 \%$ & \\
\hline
\end{tabular}

Table 5.-Summary of Prediction Errors in Column E of Original Table. 4, to Show Frequency-Distribution, Average Error and Standard-

Deviation. Standard: Bornhardt's. Series : 229 Soldifrs

\begin{tabular}{|c|c|c|c|c|c|}
\hline 1 & 2 & 3 & 4 & 5 & 6 \\
\hline $\begin{array}{l}\text { Percentage } \\
\text { Prediction } \\
\text { Error }\end{array}$ & Frequency & $\begin{array}{c}\text { Deviation } \\
\text { from } \\
\text { Arbitrary } \\
\text { Value A }\end{array}$ & $\begin{array}{c}\text { Product } \\
\text { Column } 2 \\
\times \\
\text { Column } 3\end{array}$ & $\begin{array}{l}\text { Deviation } \\
\text { Squared }\end{array}$ & $\begin{array}{c}\text { Product } \\
\text { Column } 2 \\
\times \\
\text { Column } 5\end{array}$ \\
\hline $\mathbf{E}$ & $f$ & $z$ & f. $z$ & $z^{2}$ & f. $z^{2}$ \\
\hline $\begin{array}{r}0 \\
1 \\
2 \\
3 \\
4 \\
5 \\
6 \\
6 \\
7 \\
8 \\
9 \\
10 \\
11 \\
12 \\
13 \\
14 \\
15 \\
16 \\
17 \\
18 \\
19 \\
36\end{array}$ & $\begin{array}{r}9 \\
33 \\
27 \\
25 \\
16 \\
18 \\
\cdot 4 \\
24 \\
8 \\
11 \\
5 \\
7 \\
12 \\
7 \\
6 \\
4 \\
5 \\
1 \\
4 \\
3 \\
3 \\
1\end{array}$ & $\begin{array}{r}-6 \\
\mathbf{2} \\
-4 \\
=3 \\
=2 \\
-1 \\
0 \\
1 \\
2 \\
3 \\
4 \\
5 \\
6 \\
7 \\
8 \\
9 \\
10 \\
11 \\
12 \\
13 \\
30\end{array}$ & $\begin{array}{r}-54 \\
=165 \\
=108 \\
=75 \\
=32 \\
=18 \\
\end{array}$ & $\begin{array}{r}1 \\
4 \\
9 \\
16 \\
25 \\
25 \\
36 \\
49 \\
64 \\
81 \\
100 \\
121 \\
144 \\
169 \\
900\end{array}$ & $\begin{array}{r}324 \\
825 \\
432 \\
225 \\
64 \\
18 \\
\\
8 \\
8 \\
44 \\
45 \\
112 \\
300 \\
252 \\
294 \\
256 \\
405 \\
100 \\
484 \\
432 \\
507 \\
900\end{array}$ \\
\hline Sum............. & 229 & $\ldots$ & +1 & ... & 6,027 \\
\hline Mean...... & ... & $\ldots$ & +0.0044 & $\cdots$ & 26.3 \\
\hline
\end{tabular}


Calculation of the Standard Deriation.

Method I (Yule): From the frequency distribution Table 5.

$$
\begin{aligned}
\text { S. D. } & =\sqrt{\frac{\text { Sum f. } \mathrm{z}^{2}}{\mathrm{~N}^{\top}}-\mathrm{d}^{2}} \\
& =\sqrt{\frac{6027^{-}}{229}-(0.0044)^{2}} \\
& =\sqrt{26.3-0.000019} \\
& =\sqrt{26.299981} \\
& =\sqrt{26.3} \\
& =5.13 \%
\end{aligned}
$$

Method II (Harris and Bene$\operatorname{dict}^{28}$ ): From Tables 3 and 4.

$$
\begin{aligned}
\text { S. D. } & =\sqrt{\frac{\text { Sum } E^{2}}{N}-\left[\frac{\text { Sum E }}{N}\right]^{2}} \\
& =\sqrt{\frac{\text { Sum } E^{2}}{N}-(M)^{2}} \\
& =\sqrt{\frac{14283}{229}-(6.00)^{2}} \\
& =\sqrt{62.37-36.00} \\
& =\sqrt{26.37} \\
& =5.14 \%
\end{aligned}
$$

Az'erage Error with Regard to Sign, Algebraic Mean.-When a given prediction method gives results which are on the average too high, the mean error with regard to sign will have the positive sign. ${ }^{\mathrm{s}}$ When it is too low, it will have the negative sign. Dividing the sum of the errors with regard to sign by the total number of individuals in the series in hand we have a measure of the average error in the direction of too high or too low prediction. This value is given in Tables 7 , 8, 9. It is liable to give a factitious appearance of accuracy because the plus and minus errors tend to cancel, and the algebraic mean is therefore only mentioned in passing, while great attention is given to the arithmetic mean discussed below. Before passing to that however, another measure of the direction of the error may be presented in Table 6 .

TABLE 6.-Prediction Error Tó High or Too Low

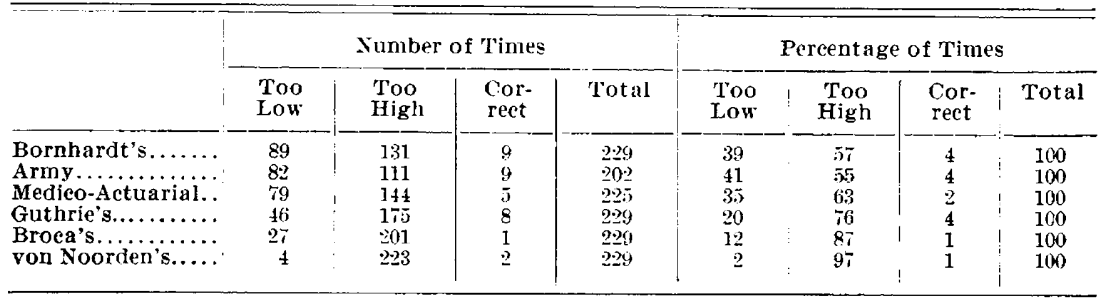

Average Error Without Regard to Sign, Arithmetic Mean.-But the question, however, as to whether a given prediction method yields on the whole too high or too low values is not the only one to be answered. One wishes to know the extent of errors, either above or below the actual weight, in the case of each of the methods used. This 
extent may be reckoned (1) by ignoring the signs and simply regarding a difference between observed and predicted body weight as an error of a given magnitude in kilograms, or better as an error of the equivalent percentage of the actual weight; then dividing the sum of these errors by the number of individuals in the series, the formula is: Sum $\mathrm{E} / \mathrm{N}=\mathrm{M}$. Another method (2) for obtaining the mean, useful as a confirmation of the above briefer method, is seen in Table 5 .

This mean error sometimes proves identical for two methods under comparison, so that further analysis by other constants is necessary. Furthermore, these other constants may be of service even in cases where they are not made absolutely necessary by similarity of means. For the arithmetical mean has two disadvantages. ${ }^{8}$ First, it does violence to sound mathematical usage with regard to signs. Second, it gives errors importance only proportional to their magnitudes, while one may consider, in a comparative test like the present, that very great errors should be given proportionally more weight than very slight deviations. Both of these drawbacks may be avoided by figuring the standard error.

Standard Error, or Root-Mean-Square Error.-In this paper this term is used in another sense than that usual among statisticians. Here, the above mentioned magnitudes of the deviations or of the percent errors may be logically weighted and the transgression against the law of signs avoided by squaring the errors, summing the squares, obtaining their mean and extracting its squares.

Range.-The error made by Bornhardt's or any other method is of course variable, and for this variation, technically called dispersion, "the simplest possible measure . . . is the actual range, i. e., the difference between the greatest and least values observed. While this is frequently quoted, it is as a rule the worst of all possible measures for any serious purpose." 32 A better measure is the standard deviation, and better still, the coefficient of variation.

Standard Deviation.-Yule ${ }^{32}$ states that "The standard deviation should always be used as the measure of dispersion, unless there is some very definite reason for preferring another measure, just as the arithmetic mean should be used as the measure of position." The standard deviation is the square root of the average of the squares of all deviations as measured from the arithmetic mean of the observations; and therefore is called the root-mean-square deviation from the mean. To square all the deviations may seem artificial, but such root-mean-square quantities frequently occur in other branches of science. The computation may be made either from the frequency Table 5, modeled on Yule, or from the briefer formula of Harris applied to figures in Table $4 .^{8,33}$

33. Harris, J. A.: Am. Naturalist 44:693, 1910. 
Coefficient of Variation.-Standard deviations are not comparable when the respective means are of different size, hence the need of translating the former from absolute to percentage values, thus securing the coefficients of variation. The formula is 100 S.D. $/$ M. = C. V.

\section{DATA COMPUTED AND ANALYZED}

Error in Relation to the Six Standards.-Our findings are summarized for the first series in Table 7 , for the second or present group in Table 8, and for the two groups consolidated in Table 9. In each table note the lower values across the first row, as compared with successive rows down the page.

TABLE 7.-Error in Relation to Standards. Summary of Prediction Errors Computed from Original of Table 4. Standards: All Six. Series: Twenty Males

\begin{tabular}{|c|c|c|c|c|c|c|c|}
\hline \multirow{5}{*}{$\begin{array}{l}\text { Rank } \\
\text { in } \\
\text { Order } \\
\text { of } \\
\text { Acels- } \\
\text { racy }\end{array}$} & \multirow{5}{*}{$\begin{array}{l}\text { Standards } \\
\text { Compared }\end{array}$} & \multirow{4}{*}{$\begin{array}{c}\text { Num- } \\
\text { ber } \\
\text { of } \\
\text { Sub. } \\
\text { jects }\end{array}$} & \multirow{2}{*}{\multicolumn{2}{|c|}{$\frac{\begin{array}{c}\text { Average or } \\
\text { Mean Error }\end{array}}{\frac{\text { Sum F }}{\mathrm{N}}}$}} & \multirow{2}{*}{$\begin{array}{c}\text { Root-Mean- } \\
\text { Square } \\
\text { Error } \\
\text { or } \\
\text { Standard } \\
\text { Error }\end{array}$} & \multirow{2}{*}{$\begin{array}{l}\text { Standard- } \\
\text { Devjation } \\
\text { of Mean } \\
\text { Error }\end{array}$} & \multirow{2}{*}{$\begin{array}{l}\text { Coeffi- } \\
\text { cient of } \\
\text { Varia- } \\
\text { tion of } \\
\text { Mean } \\
\text { Frror }\end{array}$} \\
\hline & & & & & & & \\
\hline & & & $\begin{array}{c}\text { Algebraic } \\
\text { Mean }\end{array}$ & $\begin{array}{l}\text { Arithmetic } \\
\text { Mean }\end{array}$ & & & \\
\hline & & & $\begin{array}{l}\text { Average } \\
\text { With } \\
\text { Regard } \\
\text { to Sign }\end{array}$ & $\begin{array}{l}\text { Average } \\
\text { Withont } \\
\text { Regard } \\
\text { to Sign }\end{array}$ & $\frac{\operatorname{Sum}\left(\mathrm{K}^{2}\right)}{\mathrm{N}}$ & $\frac{\operatorname{Sum}\left(\mathrm{E}^{2}\right)}{\mathrm{N}}-\mathrm{M}^{2} \mid$ & $\frac{100 \mathrm{SD}}{\mathrm{M}}$ \\
\hline & & $\mathbf{N}$ & $A$ & $\mathbf{M}$ & S.E. & S.D. & C.V. \\
\hline 1 & 2 & 3 & 4 & 5 & 6 & 7 & 8 \\
\hline $\begin{array}{l}1 \\
2 \\
3 \\
\\
4 \\
5 \\
6\end{array}$ & 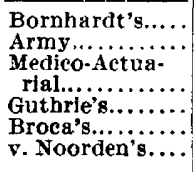 & $\begin{array}{l}20 \\
18 \\
20 \\
20 \\
20 \\
20\end{array}$ & $\begin{array}{r}-2.7 \\
+2.6 \\
+3.1 \\
+8.8 \\
+13.1 \\
+19.8\end{array}$ & $\begin{array}{r}5.6 \pm 0.542 \\
7.3 \pm 1.03 \\
7.4 \pm 0.77 \\
9.5 \pm 1.22 \\
13.1 \pm 1.37 \\
19.9 \pm 1.84\end{array}$ & $\begin{array}{r}6.6 \\
9.8 \\
\\
8.9 \\
12.5 \\
15.9 \\
23.4\end{array}$ & $\begin{array}{r}3.6 \\
6.5 \\
\\
5.1 \\
8.1 \\
9.1 \\
12.2\end{array}$ & $\begin{array}{l}64.3 \\
89.0 \\
68.9 \\
85.3 \\
69.5 \\
61.3\end{array}$ \\
\hline
\end{tabular}

Table 8.-Error in Rfiation to Standards. Standards: All Six. SERIES : 229 SOLDIERS

\begin{tabular}{|c|c|c|c|c|c|c|c|}
\hline \multirow{2}{*}{$\begin{array}{l}\text { Rank } \\
\text { in } \\
\text { Order } \\
\text { of } \\
\text { Merit }\end{array}$} & \multirow{2}{*}{$\begin{array}{l}\text { Standards } \\
\text { Compared }\end{array}$} & \multirow{2}{*}{$\begin{array}{c}\begin{array}{c}\text { Num- } \\
\text { ber } \\
\text { of } \\
\text { Cases }\end{array} \\
\mathbf{N}\end{array}$} & \multicolumn{2}{|c|}{$\begin{array}{l}\text { Averag: or } \\
\text { Mean Error }\end{array}$} & \multirow{2}{*}{$\begin{array}{l}\begin{array}{c}\text { Stain- } \\
\text { dard } \\
\text { Error }\end{array} \\
\text { S.E. }\end{array}$} & \multirow{2}{*}{$\begin{array}{l}\begin{array}{c}\text { Stan- } \\
\text { dard } \\
\text { Devia- } \\
\text { tion }\end{array} \\
\text { S.D. }\end{array}$} & \multirow{2}{*}{$\begin{array}{c}\begin{array}{c}\text { Coeffi- } \\
\text { cient. of } \\
\text { Varia- } \\
\text { tion }\end{array} \\
\text { C.V. }\end{array}$} \\
\hline & & & $A$ & $\mathbf{M}$ & & & \\
\hline $\begin{array}{l}1 \\
2 \\
3 \\
4 \\
5 \\
6\end{array}$ & 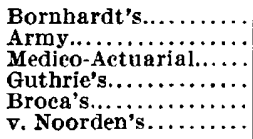 & $\begin{array}{l}229 \\
202 \\
228 \\
229 \\
229 \\
229\end{array}$ & $\begin{array}{l}+1.4 \\
+2.0 \\
+3.5 \\
+8.7 \\
+13.0 \\
+23.6\end{array}$ & $\begin{array}{r}6.0 \pm 0.228 \\
8.4 \pm 0.318 \\
8.7 \pm 0.291 \\
11.7 \pm 0.374 \\
14.7 \pm 0.418 \\
23.7 \pm 0.504\end{array}$ & $\begin{array}{r}7.9 \\
10.7 \\
10.9 \\
14.4 \\
17.4 \\
26.3\end{array}$ & $\begin{array}{r}5.1 \\
6.7 \\
6.5 \\
8.4 \\
9.4 \\
11.3\end{array}$ & $\begin{array}{l}85.0 \\
79.6 \\
74.7 \\
71.8 \\
64.0 \\
47.7\end{array}$ \\
\hline
\end{tabular}

Error in Relation to Age.-The question may be raised, do prediction errors vary proportionately with age, actual weight, height, or chest-girth (either directly, inversely, or at the extremes from the average)? The age ranged from 18 to 34 years, with an average of 
25.9 years. The mode, i. e., the most frequent or typical age, was 23 years. Eighty-six per cent. of the men were in their twenties. Ninety per cent. were 23 or over, "the age when the average man has attained his full stature and bulk," according to Quetelet, ${ }^{6}$ Dawson, Aitkin and Beddoe. ${ }^{21}$ It should be remembered that this age is taken from the nearest, not the last, birthday. The frequency distribution shows that the error is least at the average age, i. e., 26, runs high at isolated ages, but does not tend to be greater over the younger or indeed over any significant period. The errors for the extreme age groups are bracketed, as being unreliable, owing to the small number of cases in each of those classes (Table 10).

TABLE 9.-Error in Relation to Standards. Sertes: $20+229=249$ Malfs

\begin{tabular}{|c|c|c|c|c|c|c|c|c|}
\hline \multirow[t]{2}{*}{$\begin{array}{l}\text { Rank } \\
\text { jn } \\
\text { Order } \\
\text { of } \\
\text { Merit }\end{array}$} & \multirow[t]{2}{*}{$\begin{array}{l}\text { Standards } \\
\text { Compared }\end{array}$} & \multirow{2}{*}{$\left|\begin{array}{c}\text { Num- } \\
\text { ber } \\
\text { of } \\
\text { Subjects }\end{array}\right|$} & \multicolumn{2}{|c|}{$\begin{array}{l}\text { Average or } \\
\text { Mean Error }\end{array}$} & & \multirow{2}{*}{$\begin{array}{l}\text { Stan- } \\
\text { dard } \\
\text { Frror } \\
\text { S.E. }\end{array}$} & \multirow{2}{*}{$\begin{array}{l}\begin{array}{c}\text { Stan- } \\
\text { dard- } \\
\text { Devia- } \\
\text { tion }\end{array} \\
\text { S.D. }\end{array}$} & \multirow{2}{*}{$\begin{array}{l}\begin{array}{c}\text { Coeffi- } \\
\text { cient of } \\
\text { Varia- } \\
\text { tion }\end{array} \\
\text { C.V. }\end{array}$} \\
\hline & & & $A$ & $\mathbf{M}$ & & & & \\
\hline $\begin{array}{l}1 \\
2 \\
3 \\
4 \\
5 \\
6\end{array}$ & 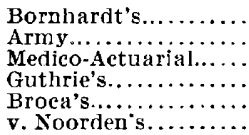 & $\begin{array}{l}249 \\
220 \\
248 \\
249 \\
249 \\
249\end{array}$ & $\begin{array}{l}+1.1 \\
+2.0 \\
+3 . \overline{3} \\
+8.1 \\
+13.0 \\
+23.2\end{array}$ & $\begin{array}{r}6.0 \pm 0.213 \\
8.3 \pm 0.304 \\
8.6 \pm 0.274 \\
11 . \overline{ \pm} 0.358 \\
14.6 \pm 0.396 \\
23.4 \pm 0.485\end{array}$ & 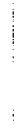 & $\begin{array}{l}7.8 \\
10.7 \\
10.7 \\
14.3 \\
17.3 \\
26.0\end{array}$ & $\begin{array}{r}5.0 \\
6.7 \\
6.4 \\
8.4 \\
9.3 \\
11.4\end{array}$ & $\begin{array}{l}83.3 \\
80.7 \\
74.4 \\
73.0 \\
63.7 \\
48.7\end{array}$ \\
\hline
\end{tabular}

TABLE 10,--Distribution of Error According to Age Standard: Bornhardt's. Series: 229 Males

\begin{tabular}{|c|c|c|c|c|c|c|c|c|c|c|c|c|c|c|c|c|c|c|}
\hline \multirow{3}{*}{$\begin{array}{l}\text { Class-frequencies, } \\
\text { i. e., number of } \\
\text { cases in each } \\
\text { class........... }\end{array}$} & \multicolumn{18}{|c|}{ Nearest Age } \\
\hline & 18 & 19 & 20 & 21 & $\mathbf{2} 2$ & 23 & 24 & 25 & 26 & 27 & 28 & 29 & 30 & 31 & 32 & 33 & 34 & Sum \\
\hline & 1 & 1 & 1 & 5 & 15 & 38 & 23 & 31 & 19 & 20 & 30 & $1 \overrightarrow{3}$ & 12 & 12 & 2 & 3 & 1 & 229 \\
\hline each age....... & $(2$ & 11 & j & 6) & 9 & 5 & 5 & 6 & 4 & 6 & $\vec{\imath}$ & 5 & 9 & 7 & $(6$ & 5 & 11) & \\
\hline
\end{tabular}

Error in Relation to Actual Weight.-.The weight varied from 47.6$87.5 \mathrm{~kg}$. (105-193 lbs.), with a mean of $64.1 \mathrm{~kg}$. (141 lbs.), and a mode of $61.2 \mathrm{~kg}$. (135 lbs.). The frequency distribution is summarized in Table 11. In choosing the magnitude of the class-interval, the frequency distribution unit of 1 pound each would have given 88 classes which would have been unwieldy and would have included too few figures in each class; units of one kilogram each with 40 classes, would have the same drawbacks; 2 kilogram intervals would have given the very desirable number of classes, ${ }^{32}$ but would still have had a rather small number of cases in each. The size chosen also has the advantage of keeping both kilos and pounds as integers. Even with only eight classes the last covers only two cases, and must be ignored in the inter- 
pretation. The error is seen to be least for the groups including the central weights, as would be expected, and to increase toward the extremes.

Error in Relation to Height.-The stature in bare feet averaged 171 $\mathrm{cm}$. (67 inches), ranging from $147-191 \mathrm{~cm}$. (58-75 inches). No correlation is seen in Table 12 .

TABLE 11.-Distribution of ERror According to Weight* Standard: Bornhardt's. Series : 229 Males

\begin{tabular}{|c|c|c|c|c|c|c|c|c|c|}
\hline Wejght........ \{ $\left\{\begin{array}{l}\mathrm{Kg} . \\
\mathrm{Lbs} .\end{array}\right.$ & $\begin{array}{l}48 \text { and } \\
\text { under } \\
53 \\
106 \text { up }\end{array}$ & $53-$ & $58-$ & $\begin{array}{l}63- \\
139-\end{array}$ & $\begin{array}{l}68- \\
150-\end{array}$ & $73-$ & 78 & $\begin{array}{l}83- \\
183-\end{array}$ & $\begin{array}{l}47.6-87.5 \\
105-193\end{array}$ \\
\hline Number of cases.... & 13 & 31 & 73 & 49 & 36 & 16 & 9 & 2 & 229 \\
\hline $\begin{array}{c}\text { Average error for each } \\
\text { weight class............. }\end{array}$ & 12 & 9 & 5 & 4 & 5 & 7 & 13 & (13) & \\
\hline
\end{tabular}

* Each class-jnterval $=5 \mathrm{~kg} .=11 \mathrm{lbs}$.

TABle 12,-Distribution of Error According to Height * Standard: Bornhardt's. Series: 229 Males

\begin{tabular}{|c|c|c|c|c|c|c|c|c|c|c|}
\hline Height.... $\left\{\begin{array}{l}\text { Cm. } \\
\text { In. }\end{array}\right.$ & $\begin{array}{l}147 \text { and } \\
\text { under } \\
152 \\
58 \text { up }\end{array}$ & $\begin{array}{l}152- \\
60-\end{array}$ & $\begin{array}{l}157- \\
62-\end{array}$ & $\begin{array}{l}162- \\
64-\end{array}$ & $\begin{array}{l}167- \\
66-\end{array}$ & $\begin{array}{l}172- \\
68-\end{array}$ & $\begin{array}{l}177- \\
70-\end{array}$ & $\begin{array}{l}182- \\
72-\end{array}$ & $\begin{array}{l}187- \\
74-\end{array}$ & $\begin{array}{l}147-191 \\
58-75\end{array}$ \\
\hline No. of cases..... & 1 & 2 & 19 & 33 & 51 & 65 & 43 & 11 & 4 & 229 \\
\hline $\begin{array}{l}\text { Average error } \\
\text { for each class.. }\end{array}$ & (2) & 11) & 5 & 8 & 5 & 6 & 5 & 6 & (7) & \\
\hline
\end{tabular}

* Each class interval $=5 \mathbf{c m} .=2$ inches.

TABLE 13.-Distribution of Error According to Chest-Girth * Standard: Bornhardt's. Series : 229 Soldiers

\begin{tabular}{|c|c|c|c|c|c|c|c|c|c|c|c|c|c|}
\hline Chest girth $\left\{\begin{array}{l}\mathrm{Cm} . \\
\text { In. }\end{array}\right.$ & $70\}$ & $\begin{array}{c}79 \text { and } \\
\text { under } \\
81.5 \\
31 \text { ip }\end{array}$ & $\begin{array}{l}81.5- \\
32-\end{array}$ & $\begin{array}{l}84-- \\
33-\end{array}$ & $\begin{array}{l}86.5- \\
34-\end{array}$ & $\begin{array}{l}89- \\
35-\end{array}$ & $\begin{array}{l}91.5- \\
36-\end{array}$ & $\begin{array}{l}94- \\
37-\end{array}$ & $\begin{array}{l}96 . \overline{5}- \\
38-\end{array}$ & $\begin{array}{l}99- \\
39-\end{array}$ & $\begin{array}{c}101.5- \\
40-\end{array}$ & $\begin{array}{l}104- \\
41-\end{array}$ & $\begin{array}{l}70-106 \\
28-42\end{array}$ \\
\hline No. of cases...... & 1 & 4 & 9 & 40 & 29 & 79 & 15 & 33 & 9 & 6 & 3 & 1 & 229 \\
\hline $\begin{array}{l}\text { Average error } \\
\text { for each class. }\end{array}$ & 36 & 3 & 8 & 6 & 5 & 6 & 7 & 6 & 5 & 4 & 5 & 2 & \\
\hline
\end{tabular}

* Class interval $=2.5 \mathrm{~cm} .=1$ inch.

Error in Relation to Chest-Girth.-The perimeter of the thorax, at the level of the mammillae, midway between inspiration and expiration, varied from 70 to $106 \mathrm{~cm}$. (from 28 to 42 inches) with an average of $90 \mathrm{~cm}$. (35 inches). No correlation is evident (Table 13). 


\section{CONCLUSIONS}

The normal weight for an American man may be predicted somewhat more accurately by Bornhardt's formula than by the Army and Navy table, the Medico-Actuarial, Guthrie's, Broca's, or Von Noorden's standard. This was true for a series of 249 native-born men, aged 18 to 34 years, inclusive. Whether it would also hold true for other men, for women and for children, we cannot state. Bornhardt's standard did not vary in accuracy at varying ages, weights, heights and chest-girths. Though other writers have urged the value of routinely recording the chest perimeter as well as height, nobody has incorporated it into a formula as simple as Bornhardt's. And, just as his rule (based on height and chest-girth) as a rough measure of surface seems both theoretically and empirically the best guide so far offered for guessing weight, so we expect that experiment would discover an even better rule (based on height, chest and a third factor) expressing a rough measure of volume. For fundamentally weight must be proportional, not to length nor surface, but to cubic mass.

We wish to express our thanks for assistance of various kinds to Prof. I. I. Lemann of New Orleans, formerly Major M. C. and chief of the medical service of the unit, to Dr. A. B. Brower of Dayton, Ohio, to Dr. F. B. Allen of North Wales, Pa., and to Prof. J. L. Coolidge of Cambridge, Mass. 